\title{
Aging-associated changes in transcriptional elongation influence metazoan longevity
}

Cédric Debès ${ }^{1 \#}$, Sebastian Grönke#, Özlem Karalay²\#, Luke Tain², Shuhei Nakamura², Oliver Hahnn,2, Carina Weigelt ${ }^{2}$, Anne Zirkel ${ }^{5}$, Konstantinos Sofiadis ${ }^{5}$, Lilija Brant ${ }^{5}$, Bernd Wollnik ${ }^{7}$, Torsten Kubacki $^{3}$, Martin Späth ${ }^{3}$, Bernhard Schermer ${ }^{1,3,4,5}$, Thomas Benzing ${ }^{1,3,4,5}$, Roman-Ulrich Müller ${ }^{1,3,4}$, Argyris Papantonis ${ }^{8^{*}}$, Adam Antebi ${ }^{2,4^{*}}$, Linda Partridge ${ }^{2,4,6^{*}}$, Andreas Beyer ${ }^{1,4,5 *}$

\section{Affiliations:}

${ }^{1}$ CECAD, University of Cologne, Faculty of Medicine and University Hospital Cologne, Cologne, Germany.

${ }^{2}$ Max Planck Institute for Biology of Aging, Joseph-Stelzmann-Str. 9b, 50931 Cologne, Germany.

${ }^{3}$ Department II of Internal Medicine and Center for Molecular Medicine Cologne, University of Cologne, Faculty of Medicine and University Hospital Cologne, Cologne, Germany.

${ }^{4}$ Systems Biology of Aging Cologne, University of Cologne, 50923 Cologne, Germany.

${ }^{5}$ Center for Molecular Medicine of the University of Cologne, 50931 Cologne, Germany.

${ }^{6}$ Institute of Healthy Aging, Department of Genetics, Evolution and Environment, UCL, London WC1E 6BT, UK.

${ }^{7}$ University Medical Center Göttingen, 37073 Göttingen, Germany.

${ }^{8}$ Institute of Pathology, University Medical Centre Göttingen, 37075 Göttingen, Germany.

\# These authors contributed equally to this work.

*Correspondence to: Email: argyris.papantonis@med.uni-goettingen.de (A.P.);

aantebi@age.mpg.de (A.A.); Partridge@age.mpg.de (L.P.); andreas.beyer@uni-koeln.de

(A.B.) 


\begin{abstract}
Aging impairs cellular homeostasis, thereby compromising multiple cellular processes, including transcription and splicing. However, the molecular mechanisms at work, and hence ways of preventing loss of transcriptional fidelity, are so far elusive. We analyzed changes in genome-wide, transcription-coupled processes with age in Caenorhabditis elegans, Drosophila melanogaster, Mus musculus, Rattus norvegicus and Homo sapiens. Using total RNA profiling, we quantified transcriptional elongation speed (Pol-II speed). Genomeaveraged Pol-II speed increased with age in all five species. Lifespan-extending dietary restriction and lowered insulin signaling both rescued these age-related trends. Experimentally reducing Pol-II speed in worms and flies increased lifespan. These findings uncover fundamental molecular mechanisms driving animal aging and underlying lifespanextending interventions, and point to possible preventative measures.
\end{abstract}

\title{
One-sentence summary
}

Genome-wide analysis of total RNA during aging reveals increased transcriptional elongation speed and decreased splicing efficiency, with an impact on longevity. 


\section{Main Text}

Aging impairs a wide range of cellular processes, many of which affect the quality and concentration of proteins. Among these, transcription is particularly important, because it is the main regulator of protein levels (1-3). Transcriptional elongation is critical for proper mRNA synthesis, due to its association with pre-mRNA processing steps such as splicing, editing, and 3' end formation $(4,5)$. Indeed, dysregulation of transcriptional elongation can lead to a number of diseases (6). During aging, animal transcriptomes undergo extensive remodeling, with large-scale changes in expression of transcripts involved in signaling pathways, DNA damage response, protein homeostasis (e.g. the ubiquitin-proteasome pathway), immune response, and stem cell plasticity (7). Further, some studies detected an age-related increase in variability and error of gene expression (8-10). This prior work has provided insights into how the transcriptome adapts to, and is affected by, aging-associated cellular stress. However, it is not known if, or to what extent, the transcription process itself is affected by aging. In this study, we have investigated how the kinetics of transcription is affected by aging, how such changes can affect mRNA biosynthesis, and the role of these changes in age-related loss of function in the whole organism.

The translocation speed of RNA polymerase II (Pol-II) during transcription can be monitored using total RNA sequencing (RNA-seq), because the distribution of reads in introns is affected by the elongation speed of Pol-II. Co-transcriptional splicing results in a characteristic saw-tooth pattern of the read coverage, observable from total RNA-seq or nascent RNA-seq measurements $(11,12)$. The read coverage generally decreases 5' to 3' along an intron and the extent of this decrease depends on Pol-II speed: the faster the elongation, the shallower the slope (13-15), i.e. fast elongation of Pol-II results in fewer nascent transcripts interrupted inside introns at the moment when the cells are lysed (Fig. 1, A and B). Thus, by quantifying the gradient of read coverage along an intron it is possible to 
determine Pol-II elongation speeds at the resolution of single introns (Fig. 1, A and B). Note that this measure is only weakly associated with the expression level of the transcript (16) (Fig. S1).

To monitor the kinetics of transcription, we quantified the distribution of intronic reads resulting from RNA sequencing in five animal species: the worm C. elegans, the fruit fly $D$. melanogaster, the mouse $M$. musculus, the rat $R$. norvegicus (17), and human $H$. sapiens, at different adult ages (Extended Data Table 1, see Methods). Samples were taken from diverse mammalian tissues (brain, liver, kidney, whole blood), from fly brains, and whole worms.

Human samples originated from whole blood (healthy donors, age 21-70), fibroblasts from an individual with a progeria syndrome compared to an age- and sex-matched healthy control, and from two human cell lines (IMR90, HUVEC) that were driven into replicative senescence. After filtering, we obtained between 546 and 14,593 introns that passed the quality criteria for reliable Pol-II speed quantification (see Methods). Interestingly, we observed an increase of average Pol-II rates with age for all five species and all tissue types examined (Fig. 1C and Fig. S2). The average effect sizes varied from $+33 \mathrm{bp} / \mathrm{min}$ in mouse kidney to $+146 \mathrm{bp} / \mathrm{min}$ in rat liver. To address whether the lifespan-extending interventions inhibition of insulin/insulin-like growth factor signaling (IIS) and dietary restriction affected Pol-II speeds, we sequenced samples from IIS mutants, using daf-2 mutant worms at day 14 and fly brains from dilp2-3,5 mutants at day 30 and day 50, and hypothalamus from aged wild type and IRS1 null mice. Further, we sequenced samples from kidney and liver of dietary restricted (DR) and ad libitum fed mice. In all comparisons, the lifespan-extending interventions resulted in a significant reduction of Pol-II speed. Pol-II elongation speed thus increased with age across a wide range of animal species and tissues, and this increase was at least partially reverted under various lifespan-extending conditions. 

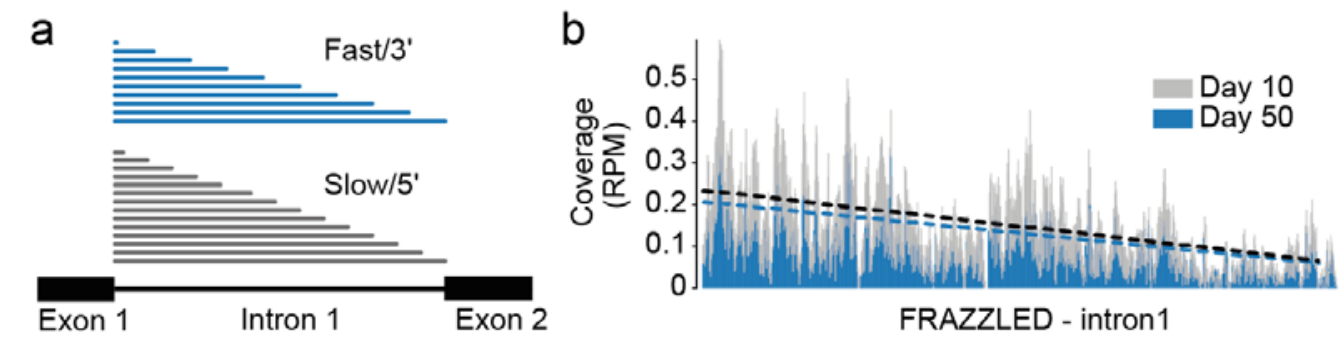

C

"old" vs "young"

"ageing intervention" vs "control"

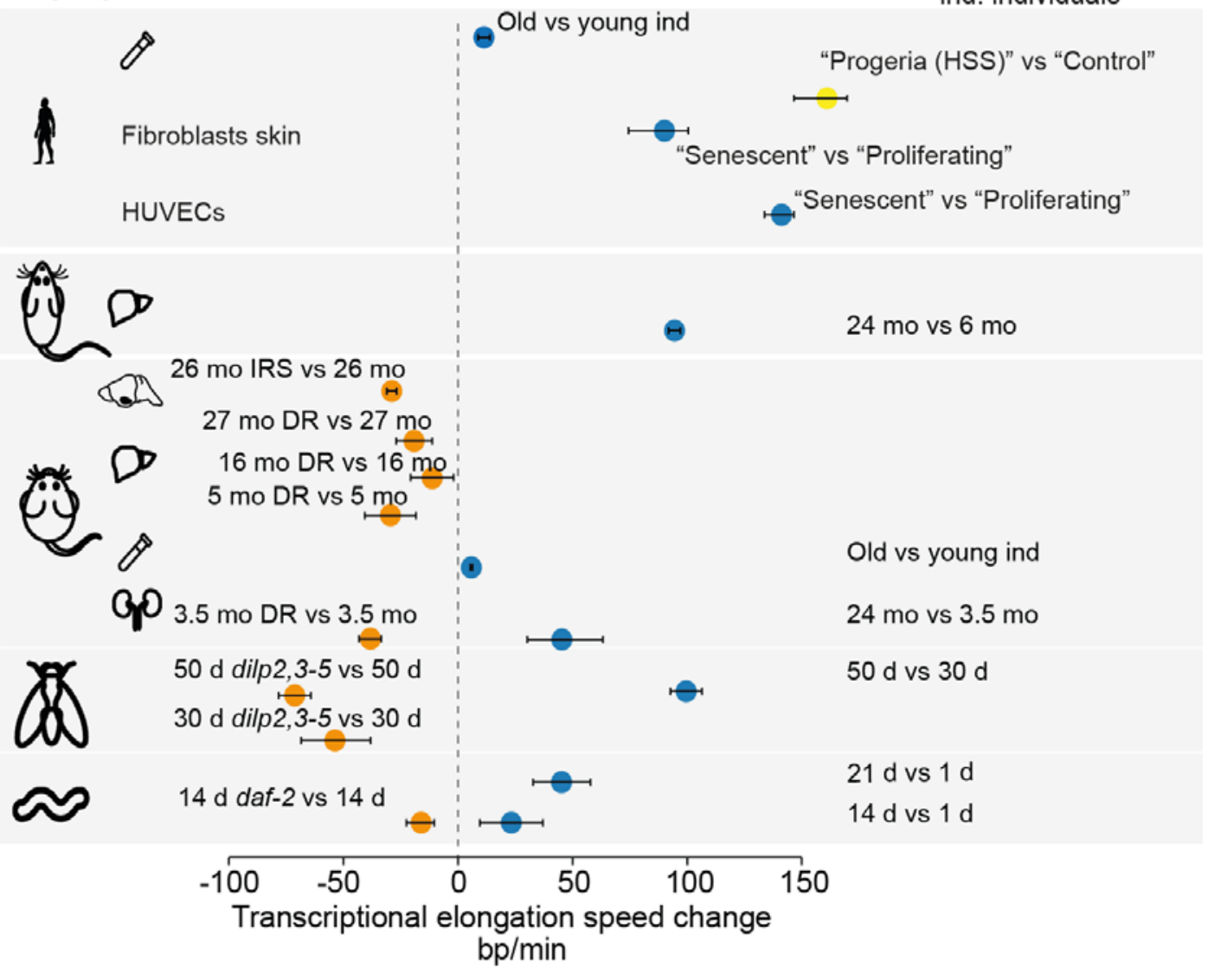

Fig. 1: Pol-II elongation rate increases with age and is slowed-down by reduced insulin signaling and dietary restriction (DR) in multiple species. (A) Schematic representation of read coverage along introns in total RNA seq. Intronic reads represent transcriptional production at a given point in time. A shallower slope of the read distribution is a consequence of increased Pol-II elongation speed. (B) Exemplary read distribution in the FRAZZLED intron 1 with coverage in RPM for D. melanogaster at age day 10 (grey) and day 50 (blue). (C) Change of average Pol-II elongation rates ( $\mathrm{bp} / \mathrm{min}$ ) in worm (whole body), fruit fly (brains), mouse (kidney, liver, hypothalamus, blood), rat (liver), human blood, and human cell culture (Fibroblast skin cells derived from a HSS progeria patient compared to a healthy control; HUVECs: umbilical vein endothelial; IMR90: fetal lung). Error bars show median variation $\pm 95 \%$ confidence interval. All changes of average elongation speeds are significantly different from zero $(\mathrm{P}<0.001$; paired Wilcoxon rank test). Number of introns considered $(n)$ ranged from 546 to 14593. 
To determine whether genes with particular functions were more strongly affected by Pol-II speed changes, we performed gene set enrichment analysis on the 200 genes with the highest increase in Pol-II speed during aging in worms, fly brains, mouse kidneys and livers and rat livers. Only very generic functional classes, such as metabolic activity, were consistently enriched across three or more species (see Methods and Fig. S3). Thus, no specific cellular process seemed to be affected consistently across species and tissues. Next, we examined age-associated gene expression changes of transcription elongation regulators. We observed that some regulators (PAF1, THOC1) were consistently down-regulated across species during aging (Fig. S4), which was also confirmed using gene set enrichment analysis (Fig. S5).

These findings suggest a potential cellular response to unwanted variation of transcriptional elongation speed.

To determine if changes in Pol-II elongation speed were causally involved in in the aging process, we used genetically modified worm and fly strains carrying point mutations in a PolII main subunit that reduce its elongation speed (C. elegans, ama-1 (m322) mutant (18) ; D. melanogaster, RpII215 ${ }^{C 4}$ mutant (19)). To confirm that these mutants reduced Pol-II elongation speed, we sequenced total RNA from wild type and slow Pol-II mutant worms (whole body at day 14) and fly heads, at day 10 and day 50. Estimates of transcription elongation rate confirmed the effects of the Pol-II slow mutants: average Pol-II speeds were lower in C. elegans ama-1 (m322) by -78 bp/min and in D. melanogaster RpII215 ${ }^{C 4}$ by -70 bp/min (Fig. 3A). To determine if Pol-II elongation rate, with its associated maintenance of transcriptional fidelity, also affected aging of the whole organism, we measured effects on lifespan. Slowing down Pol-II resulted in increased lifespans in both the worm and fruit fly (median lifespan increase: C. elegans: 20 \%; D. melanogaster: 10 \%; Fig. 2B and Fig. S6). Further, mutant worms had a higher pharyngeal pumping rate at older age compared to wild 
type worms, suggesting that healthspan was also extended by slowing down Pol-II elongation speed (Fig. S7).
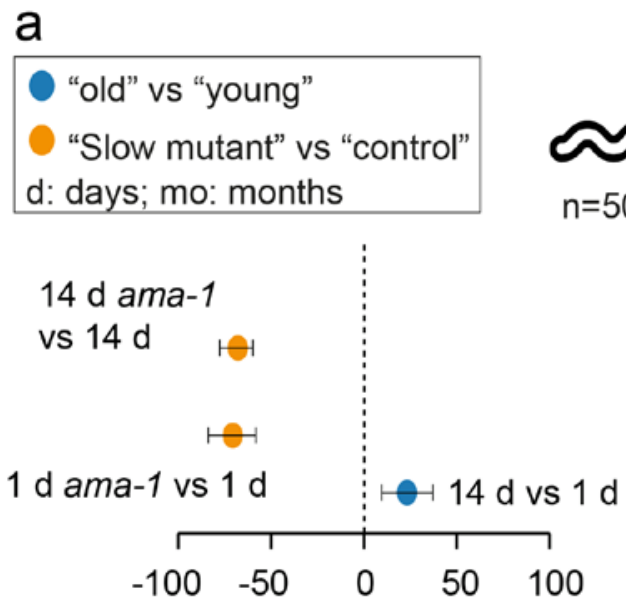

Transcriptional elongation speed change $b p / \min$

b

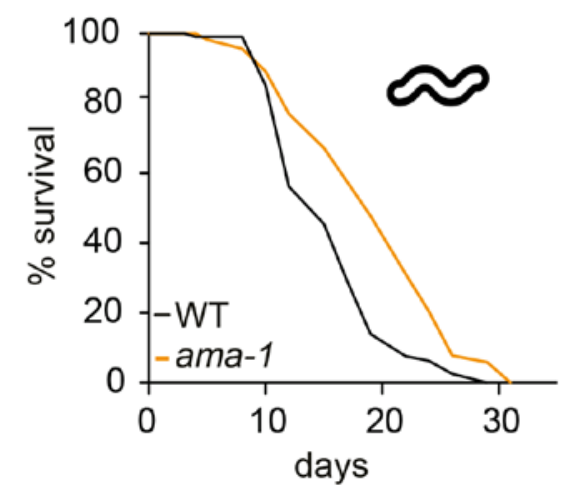

\section{$50 \mathrm{~d} R p / 1215^{\mathrm{c} 4}$}

vs $50 \mathrm{~d}$ |⿵

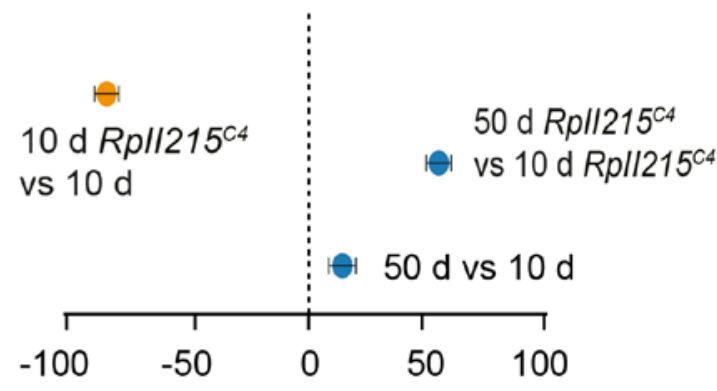

Transcriptional elongation speed change $\mathrm{bp} / \mathrm{min}$

Fig. 2: Molecular and lifespan effects of reduced Pol-II elongation speed in C. elegans and D.

melanogaster. (A) Differences of average Pol-II elongation speeds between Pol-II mutant and wild-type worms (left) and flies (right), and changes of average Pol-II elongation speeds with age in flies (right). Error bars show median variation $\pm 95 \%$ confidence interval. All average changes of Pol-II elongation speeds are significantly different from zero (P < 0.001; paired Wilcoxon rank test). (B) Survival curves of animals with RpII215 C4 mutation and wild-type controls for worms (left, replicate 1) and flies (right, averaged survival curve) (worms 4 replicates, See Fig. S4; flies 3 replicates). Animals with slow Pol-II have a significantly increased lifespan (+20\% and +10\% median lifespan increase for C. elegans ( $\mathrm{n}=120, \mathrm{P}<0.001$, log rank test) and $D$. melanogaster ( $\mathrm{n}=220, \mathrm{P}<0.001$, log rank test), respectively). 
An optimal elongation rate is required for fidelity of alternative splicing $(20,21)$ : for some exons, slow elongation favors weak splice sites, leading to exon inclusion, while these are skipped during fast elongation $(5,22,23)$. Further, a faster elongation rate can promote intron retention, leading to degradation of the transcript via nonsense-mediated decay (NMD) (24) or inactivation of tumor suppressor effects (25). Therefore, we next quantified changes in splicing efficiency as the fraction of spliced reads from all reads aligning over a given splice site (26). In most datasets from total RNA and nascent RNA sequencing we observed an increase of the spliced exon junctions relative to unspliced junctions during aging and a reduction of the percent spliced junctions under lifespan-extending conditions (Fig. S8. Thus, surprisingly we observed more spliced transcripts under conditions when Pol-II speed is accelerated, i.e. greater splicing efficiency. In the case of co-transcriptional splicing, the removal of lariats depends on the position of Pol-II in the gene $(27,28)$. In this context, our data therefore suggests that accelerated transcription shortens the time to splicing, which reduces the effective half-life of lariats.

Accelerated transcription and splicing carries the risk of increasing the number or erroneous splicing events, which in turn has been associated with advancing age and shortened lifespan (29-32). It is non-trivial to tell if a specific splice isoform is the product of erroneous splicing or if it was created in response to a specific signal. Simply checking if an observed isoform is reported in some genome annotation database is problematic for multiple reasons. On the one hand, such databases were mostly created using data from young or embryonic animals. Thus, the detected isoform may be functionally relevant in old animals, but not reported in such database. On the other hand, an annotated isoform might be the result of erroneous splicing if otherwise it should not be expressed in that particular age class or cellular context. Hence, we based our analysis on the notion that extremely rare isoforms (rare with respect to all other isoforms of the same gene in the same sample) are more likely erroneous than are 
bioRxiv preprint doi: https://doi.org/101101/719864; this version posted August 8, 2019. The copyright holder for this preprint (which was

not certified by peer review) is the author/funder, who has granted bioRxiv a license to display the preprint in perpetuity. It is made available under aCC-BY-NC-ND 4.0 International license.

frequent isoforms $(33,34)$. We defined rare splicing events as all exon-exon junctions that are supported by fewer or equal to 0.07 percent of all junctions detected for a given gene (33). Then, the gene-specific fraction of rare exon-exon junctions was computed as the number of rare exon-exon junctions divided by the total number of detected exon-exon junctions from that gene. Indeed, we observed that these rare exon-exon junctions often resulted from exon skipping or from the usage of cryptic splice sites (Fig. S9a). We observed an increase of the average fraction of rare splicing events during aging across all five species and a reversion of this effect under most lifespan-extending conditions (Fig. 3, Fig. S10). Importantly, the fraction of rare splicing events was also reduced in old flies and worms with mutations slowing down Pol-II (Fig. 3). Further, we noticed that genes with increased Pol-II elongation speed had on average larger fractions of rare isoforms than genes with reduced speed (Fig. S9b).

Another potential indicator of erroneous splicing is the increased formation of circular RNAs (circRNAs) $(35,36)$. Increased Pol-II elongation speed has previously been associated with increased expression of circRNAs (37), i.e. back-spliced transcripts with a covalent binding of their 5' and 3' ends (38). We quantified the fraction of circRNAs as the number of backspliced fragments normalized by the sum of back-spliced fragments and linearly spliced fragments. During aging (old versus young) we observed either increased or unchanged average circRNA fractions (Fig. S11). In contrast, reducing Pol-II speed reduced circRNA formation, thereby maintaining the proper splicing of genes hosting circRNAs. Thus, our data suggests that faster Pol-II elongation resulted in an increase of circRNA formation, which lead to a global increase of exon skipping for genes hosting circular RNAs. Taken together, these findings suggest that an age-associated acceleration of transcription and splicing leads to increased splicing noise with impact on lifespan. 
Interestingly, specific genes with important functions in aging-associated phenotypes were consistently affected by the changes in Pol-II elongation speed and splicing outcome in multiple species. For example, we observed an increase in transcriptional elongation speed in the DNA repair gene PARP1 during aging in human cell lines (IMR90), mouse kidney, rat liver, and fly brain (in the remaining samples the elongation speed in PARP1 could not be quantified due to insufficient read coverage in the introns). Interestingly, we observed a reduction of the major PARP1 isoform in older mammals/senescent human cells along with an increase of truncated isoforms containing only parts of the protein (Fig. S12, PARP1 and ITPR1 isoform fraction change during aging). Thus, the age-associated increase in elongation speed of PARP1 coincides with the expression of non-functional isoforms (39).

Increased Pol-II elongation speed can lead to elevated transcriptional errors, because the proofreading capacity of Pol-II is reduced (8). To explore the potential impact of transcriptional elongation on transcript quality beyond splicing, we measured the number of mismatches in aligned reads for each gene normalized by its respective expression level. During this counting, we excluded mismatches that were likely due to genome variants or other artifacts (see Methods for details). We observed that the average fraction of mismatches increased with age and that it decreased under most lifespan-extending treatment (Fig S13). Consistent with prior findings (8), slow Pol-II mutants exhibited reduced numbers of mismatches compared to their wild-type controls in 3 out 4 comparisons. Hence, our findings suggest that increased Pol-II elongation speed results in increased errors in transcripts, presumably due to a reduced proofreading capacity (8). 


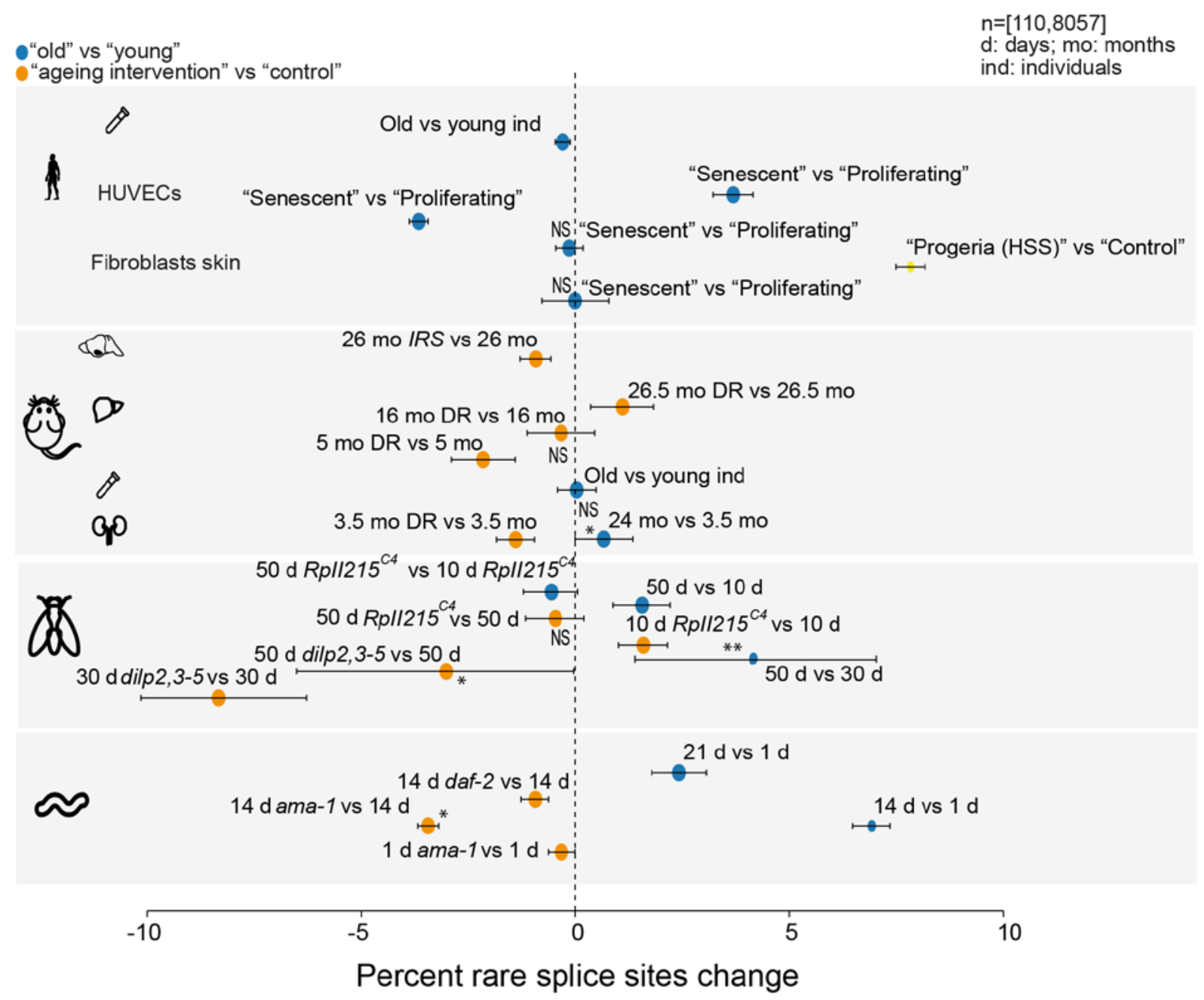

Fig. 3: Percent of rare splicing events mostly increases with age and goes down upon lifespan-extending treatments. Average percent changes of rare splice events ( $<=0.07$ percent of total gene expression) with age (blue) and upon lifespan extending treatments (orange). Error bars show median variation $\pm 95 \%$ confidence interval. All changes of average rare splice events are significantly different from zero $(\mathrm{P}<0.001$; paired Wilcoxon rank test), except those labeled NS (not significant), **P $<0.01, \quad *$ : $\mathrm{P}<0.05$. Number of genes considered ( $n$ ) ranged from 110 to 8057.

Finally, we explored alterations in chromatin structure as a potential cause of age-associated changes of Pol-II elongation speed. Nucleosome positioning along the DNA fiber is known to affect both Pol-II elongation speed and splicing efficiency (15, 40-42). Furthermore, older eukaryotic cells display reduced nucleosomal density in chromatin and “noisier” core nucleosome positioning $(43,44)$. Thus, age-associated changes in chromatin structure might contribute to the changes in Pol-II elongation speed and splicing efficiency that we observed 
here. To probe this hypothesis, we performed micrococcal nuclease (MNase) digestion of chromatin from early (proliferating) and late-passage (senescent) human IMR90 cells, followed by 400 million paired-end read sequencing of the mononucleosomal DNA fragments (MNase-seq). Following mapping, we explored changes in nucleosome occupancy and positioning over exons and exon-intron boundaries (45). We observed that nucleosome peaks moved away from exon centers and from exon-intron boundaries in senescent versus proliferating cells (Fig. 4, A and B). In addition, we quantified 'fuzziness', which reflects the precision of nucleosome positioning in a given MNase-seq dataset (see Methods). We also used the distance between nucleosome summits as a measure of the nucleosome position regularity $(46,47)$. Both measures changed significantly in senescent cells (Fig. 4, C and D): the average fuzziness was increased (in exons and introns) and the distances between nucleosome peaks became more variable in senescent cells. Finally, we compared changes in Pol-II elongation speed to fuzziness and distance. Introns showing faster transcriptional elongation exhibited greater changes in nucleosome positioning precision and regularity than introns with reduced elongation speed (Fig. 4, E and F). Importantly, whereas the average distance between nucleosomes did not differ between 'fast' and 'slow' introns, there was a significant increase of the variability of nucleosome distances in introns with increased elongation speed (Fig. 4F). These results suggest that reduced precision in the assembly of the chromatin fiber may contribute to changes in Pol-II speed and splicing fidelity (40, 44, 48) .

We have found a consistent increase in average Pol-II elongation speed with age across four animal models and three human cell lines, and we could revert this trend by employing lifespan-extending treatments. We observed consistent changes in splicing and transcript quality that correlated with Pol-II elongation speed changes and which likely contribute to 
age-associated phenotypes. Although the average changes were significant in all species, they remained small in absolute terms. This is expected as drastic genome-wide changes of RNA biosynthesis would likely lead to an immediate and complete loss of cellular functions and thus early death. Instead, here we are monitoring the gradual reduction of cellular fitness that is characteristic for normal aging. Importantly, we were able to improve splicing quality and lifespan in two species by reducing the Pol-II elongation speed. Thus, despite being small in magnitude, the effects are clearly biologically relevant for lifespan. Whereas our work establishes altered mRNA biosynthesis as a mechanism driving aging, we still lack a complete understanding of the molecular mechanisms driving Pol-II elongation speed changes.

Our work established that the elongation speed of Pol-II as an important contributor to molecular and physiological traits with implications beyond aging. Mis-regulation of transcriptional elongation factors reduces cellular and organismal fitness and may therefore contribute to disease phenotypes (49). Taken together, the data presented here reveal a new molecular mechanism contributing to aging and serve as a template for assessing the fidelity of cellular machinery during aging and disease. 

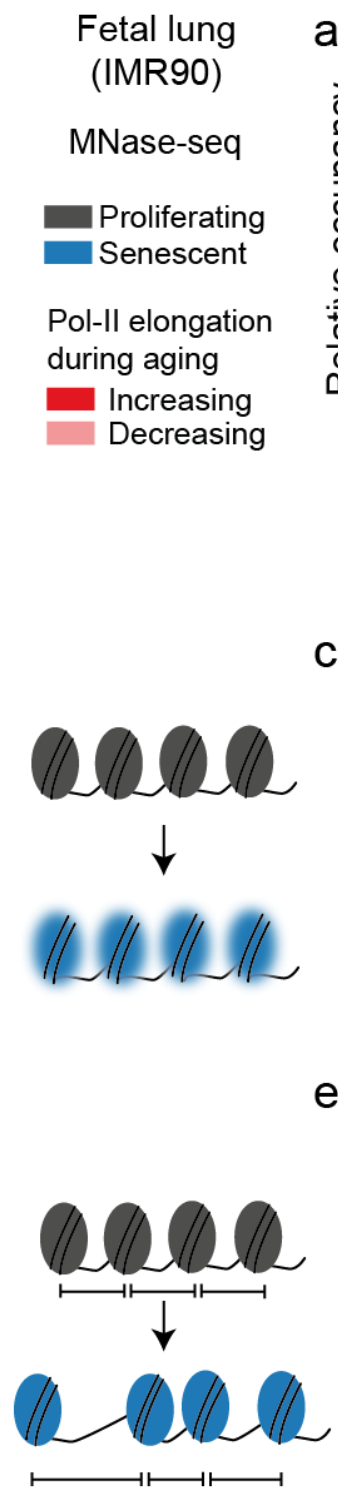
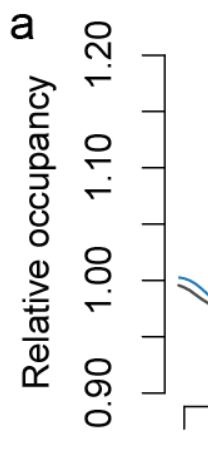
$-300$

200

Genomic position relative to exons center

C
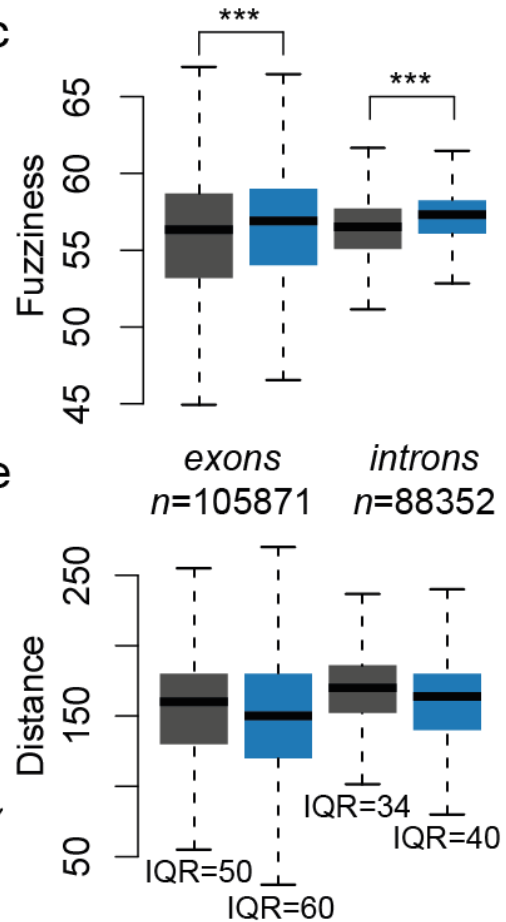

b

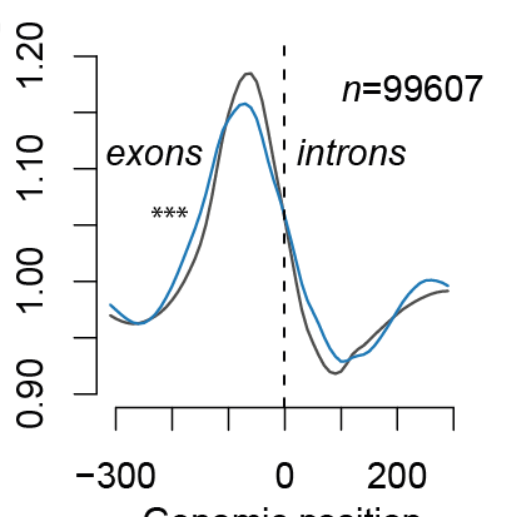

Genomic position relative to exon-intron junctions

d

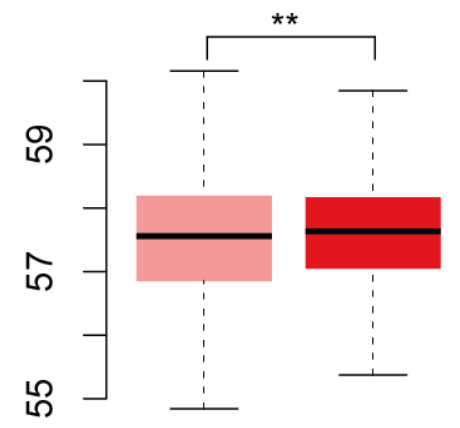

f $n=4330 \quad n=10263$

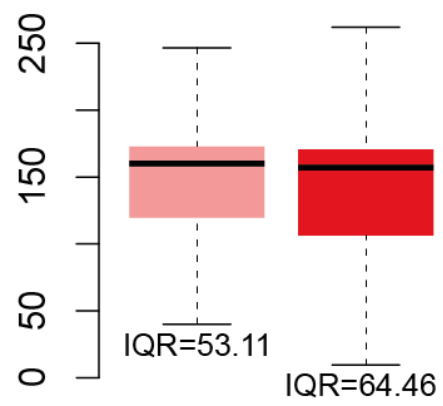

Fig. 4: Profiling of nucleosome positioning in human cell models. Meta-analysis profile of nucleosome occupancy on DNA (non-stranded) over (A) exon centers and (B) at exon-intron boundaries. Distribution of nucleosome fuzziness for (C) proliferating (grey) and senescent (dark blue) cells, and (D) increasing (dark red) and decreasing (light red) Pol-II speed. Statistical significance from difference in distribution indicated by asterisks (*** $\mathrm{P}<0.001, * * \mathrm{P}<0.01$, un-paired Wilcoxon rank test) for $\mathbf{C}$, D. Distributions of distances between nucleosome summits for (E) proliferating (grey) and senescent (dark blue), and (F) increasing (dark red) and decreasing (light red) Pol-II speed. E: nucleosomes are distinguished based on whether they are in exons (left) or introns (right). Interquartile range (IQR) is a measure of statistical dispersion E, F. 


\section{Methods}

\section{Worm strains and demography assays}

Nematodes were cultured using standard techniques at $20^{\circ} \mathrm{C}$ on NGM agar plates and were fed with E.coli strain OP50. DR786 strain carrying the ama-1(m322) IV mutation in the large subunit of Pol-II (RBP1), which confers alpha-amanitin resistance, was obtained from Caenorhabditis Genetics Center (CGC) $(50,51)$. DR786 strain was then outcrossed into N2 wild type strain 4 times and mutation was confirmed by sequencing. 5'-3' AGAAGGTCACACAATCGGAATC primer was used for sequencing. For each genotype, minimum of 120 age-matched day 1 young adults were scored every other day for survival and transferred to new plates to avoid starvation and carry-over progeny. Worms undergoing internal hatching, protruding vulva or crawling off the plates were censored. Demography experiments were repeated multiple times. For all experiments, genotypes were blinded. Statistical analyses were performed using Mantel-Cox log rank method.

\section{Fly strains and fly maintenance}

The RpII215 ${ }^{C 4}$ fly strain (RRID:BDSC_3663), which carries a single point mutation (R741H) in the gene encoding the Drosophila RNA polymerase II 215kD subunit (RBP1) was received from the Bloomington Drosophila Stock Center (Bloomington, Indiana, USA). Flies carrying the RpII215 ${ }^{C 4}$ allele (52) are homozygous viable but show a reduced transcription elongation rate (19). RpII215 ${ }^{C 4}$ mutants were backcrossed for 6 generations into the outbred white Dahomey (wDah) wild type strain. A PCR screening strategy was used to follow the RpII215 ${ }^{C 4}$ allele during backcrossing. Therefore, genomic DNA from individual flies was used as a template for a PCR reaction with primers SOL1064 (CCGGATCACTGCTGCATATTTGTT) and SOL1047 (CCGCGCGACTCAGGACCAT). The 582 bp PCR product was restricted with BspHI, which specifically cuts only in the 
RpII215 ${ }^{C 4}$ allele resulting in two bands of $281 \mathrm{bp}$ and $300 \mathrm{bp}$. At least 20 individual positive female flies were used for each backcrossing round. Long-lived insulin mutant flies, which lack three of the seven Drosophila insulin-like peptides, dilp2-3,5 mutants (RRID:BDSC_30889) (53) were also backcrossed into the wDah strain, which was used as wild type control in all fly experiments. Flies were maintained and experiments were conducted on $1,0 \mathrm{SY}$-A medium at $25^{\circ} \mathrm{C}$ and $65 \%$ humidity on a $12 \mathrm{~L}: 12 \mathrm{D}$ cycle (53).

\section{Pharyngeal pumping rate}

Synchronized wild type and ama-1 (m322) animals were placed on regular NGM plates seeded with OP50 bacteria on day1 and day8 adulthood and number of pharyngeal pumping rate was assessed by observing the number of pharyngeal contractions during a 10sec interval using dissecting microscope and Leica Application Suite X imaging software. Pharyngeal pumping rate was then adjusted for number of pharyngeal pumping per minute. Animals that displayed bursting, internal hatching and death were excluded from the experiments.

Experiments were repeated three independent times in a blinded fashion, scoring minimum of 15 randomly selected animals per genotype and time point for each experiment. One-way Anova with Tukey’s multiple comparison test was used for statistical significance testing. pvalue $<0.0001^{* * * *}$, error bars represent standard deviation.

\section{Fly lifespan assays}

For lifespan assays fly eggs of homozygous parental flies were collected during a $12 \mathrm{~h}$ time window and the same volume of embryos was transferred to each rearing bottle ensuring standard larval density. Flies that eclosed during a $12 \mathrm{~h}$ time window were transferred to fresh bottles and were allowed to mate for $48 \mathrm{~h}$. Subsequently, flies were sorted under brief $\mathrm{CO}_{2}$ anesthesia and transferred to vials. Flies were maintained at a density of 15 flies per vial and were transferred to fresh vials every two to three days and the number of dead flies was 
counted. Lifespan data were recorded using Excel and were subjected to survival analysis (log rank test) and presented as survival curves.

\section{Mouse maintenance and dietary restriction protocol}

The DR study was performed in accordance with the recommendations and guideline of the Federation of the European Laboratory Animal Science Association (FELASA), with all protocols approved by the Landesamt für Natur, Umwelt und Verbraucherschutz, NordrheinWestfalen, Germany. Details of the mouse liver DR protocol have been published previously (Hahn et al., 2017). For the mouse kidney, Male C57BL/6 mice were housed under identical SPF conditions in group cages (5 or fewer animals per cage) at a relative humidity of $50-60 \%$ and a 12 hour light and 12 hour dark rhythm. For dietary restriction vs control, 8 week old mice were used. Dietary restriction was applied for 4 weeks. Control mice received food and water ad libitum. Mice were sacrificed at 12 weeks. For comparison of young vs aged mice, 14 week and 96 week old mice were used. Food was obtained from ssniff (Art. V1534-703, Soest, Germany) and Special Diet Services (Witham, UK). The average amount of food consumed by a mouse was determined by daily weighing for a period of two weeks and was on average 4,3 g per day. DR was applied for 4 weeks by feeding $70 \%$ of the measured ad libitum amount of food. Water was provided ad libitum. Mice were weighed weekly to monitor weight loss. Neither increased mortality nor morbidity was observed during dietary restriction.

\section{RNA extraction}

Wild-type N2 strain, alpha-amanitin resistant ama-1(m322) mutants and long-lived insR/IGF signaling mutants, daf-2(e1370) were sent for RNA-seq. For each genotype, more than 300 aged-matched adult worms at desired time points were collected in Trizol (Thermo Fisher Scientific, USA) in 3 biological replicates. Total RNA was extracted using RNAeasy Mini kit 
(Qiagen, Hilden, Germany). For fly samples, brains of 30 days and 50 days old dilp2-3,5 and $w$ Dah control flies were dissected manually and snap frozen. 10 days and 50 days old RpII215 ${ }^{C 4}$ mutants and wDah control flies were snap frozen and fly heads were isolated by vortexing and sieving on dry ice. Total RNA from three biological samples per treatment group was prepared using Trizol Reagent according to the manufacturer’s instructions, followed by DNAse treatment with the TURBO DNA-free Kit (Thermo Fisher Scientific). Mouse liver samples were isolated from 5, 16 and 27 months old ad libitum and DR animals, which corresponded to 2, 13 and 24 months of DR treatment, respectively. RNA was isolated by Trizol and DNase-treated. The RNA-seq data for 5 and 27 months old liver DR samples have been published previously (54), while the 16 months data are first published here. RNeasy mini Kit and Trizol were used to isolate RNA from snap-frozen kidneys as per manufacturer's instructions. Hypothalamus tissue of long-lived insulin receptor substrate 1 (IRS1-/-) knock out mice (55) and C57BL/6 black control animals was dissected manually at the age of 72 weeks. RNA was isolated by Trizol with subsequent DNase treatment. For blood samples globin RNA was removed using GLOBINclearTM Kit, mouse/rat/human, for globin mRNA depletion.

\section{Human cell culture}

Human umbilical vein endothelial cells (HUVECs) from pooled donors (Lonza, Cologne, Germany) and Human fetal lung (IMR-90) cells (from two donors) were grown to 80\% to 90\% confluence in endothelial basal medium 2-MV with supplements (EBM; Lonza) and 5\% foetal bovine serum (FBS) and MEM (Sigma-Aldrich) with 20 FBS (Gibco) and 1\% nonessential amino acids (Sigma-Aldrich) for HUVECs and IMR-90 respectively. 


\section{Total RNA and nascent RNA sequencing}

From $1 \mu \mathrm{g}$ input of total RNA, ribosomal RNA was removed using the Ribo-Zero Human/Mouse/Rat kit (Illumina). Sequencing libraries were generated according to the TruSeq stranded total RNA (Illumina) protocol. To generate the final cDNA library, products were purified and amplified by PCR for 15 cycles. After validation and quantification of the library on an Agilent 2100 Bioanalyzer, equimolar amounts of libraries were pooled. Pools of 5 libraries were sequenced per lane on an Illumina HiSeq 4000 sequencer. For a description of all the RNA-seq datasets used in this study see Extended Data Table 1. The same protocol was used to sequence cDNA libraries from human cell "factory” RNA, which was isolated as described previously (56).

\section{RNA-seq alignments and gene expression analysis}

Raw reads were trimmed with trimmomatic version 0.33 (57) using parameters 'ILLUMINACLIP:./Trimmomatic-0.33/adapters/TruSeq3-PE.fa:2:30:10 LEADING:3 TRAILING:3 SLIDINGWINDOW:4:15 MINLEN:45’ for paired-end datasets and 'ILLUMINACLIP:./Trimmomatic-0.33/adapters/TruSeq3-SE.fa:2:30:10 LEADING:3 TRAILING:3 SLIDINGWINDOW:4:15 MINLEN:45’ for single-end datasets. Alignment was performed with STAR version 2.5.1b (58) using the following parameters: '-outFilterType BySJout --outWigNorm None’ on the genome version mm10, rn5, hg19, dm6, ce5 for M. musculus, $R$. norvegicus, H. sapiens, D. melanogaster, C. elegans, respectively. We estimated transcript counts using Kallisto version 0.42.5 for each sample. To determine differentially expressed genes we used DESeq2 version 1.8.2 (59) with RUVr normalization version 1.6.2 (60). For the differential analysis of transcriptional elongation regulators, we downloaded the list of positive and negative regulators from the GSEA/MSigDB (61). Gene ontology (GO) term enrichment analysis of differentially expressed genes or genes with 
increased RNA-Pol-II elongation speed was carried out using TopGO version 2.20.0. For GO enrichment analysis of differentially expressed genes, we identified 4784 genes as evolutionarily conserved from each species of our study to humans: genes were either direct orthologues (one2one) or fusion genes (one2many) of $H$. sapiens were retrieved from ENSEMBL database using biomaRt 2.24.1 (62). Using our 4784 genes evolutionary conserved, we further divided into consistently up-regulated or down-regulated genes across species during aging or 'aging intervention' (as target set for GO enrichment: aging upregulated: 92 genes; aging down-regulated: 71; ‘aging intervention’ up-regulated: 164 genes; ‘aging intervention’ down-regulated: 473 genes; as background set 4784 orthologue genes between $R$. norvegicus, M. musculus, D. melanogaster, C. elegans, and H. hsapiens). For GO enrichment analysis of genes harboring increasing Pol-II speed, we used as target set the top 200 or 300 genes with an increase in Pol-II speed change for each species. For circular RNA, we aligned the read using STAR version 2.5.1b (58) with the following parameters:' -chimSegmentMin 15 --outSJfilterOverhangMin 15151515 --alignSJoverhangMin 15 -alignSJDBoverhangMin 15 --seedSearchStartLmax 30 --outFilterMultimapNmax 20 -outFilterScoreMin 1 --outFilterMatchNmin 1 --outFilterMismatchNmax 2 --chimScoreMin 15 --chimScoreSeparation 10 --chimJunctionOverhangMin 15’. We then extracted back spliced reads from STAR chimeric output file and normalized the number of back spliced reads by the sum of back spliced $\left(B S_{i}\right)$ and spliced reads from linear transcripts $\left(S 1_{i}, S 2_{i}\right)$ for an exon $i(7)$ :

$$
\operatorname{CircRatio}_{i}=\frac{B S_{i}}{B S_{i}+\frac{S 1_{i}+S 2_{i}}{2}} * 100
$$

Here, $S 1_{i}$ refers to the number of linearly spliced reads at the 5 ' end of the exon and $S 2_{i}$ refers to the respective number of reads at the 3 ' end of the exon. Thus, this score quantifies the percent of transcripts from this locus that resulted in circular RNA. Finally, we quantified the 
significance of the average change in circular RNA formation between two condition using

\section{the Wilcoxon rank test.}

\section{Definition of intronic regions}

All annotation files for this analysis were downloaded from Ensembl website (64) using genome version ce10 for Caenorhabditis elegans, mm10 for Mus musculus, hg19 for Homo sapiens, rn5 for Rattus norvegicus, dm6 for Drosophila melanogaster. The following filtering steps were applied on the intronic ENSEMBL annotation files: First we removed overlapping regions between introns and exons in order to avoid confounding signals due to variation in splicing or transcription initiation and termination. Overlapping introns were merged to remove duplicated regions from the analysis. In the next step we used STAR (58) to detect splice junctions and compared them to the intronic regions. Introns with at least 5 split reads bridging the intron (i.e. mapping to the flanking exons) per condition were kept for subsequent analyses. Thereby we ensured a minimum expression level of the spliced transcript. When splice junctions were detected within introns, we further subdivided those introns accordingly. Introns with splice junction straddling were discarded. The abovementioned steps were performed using Bedtools version 2.22.1 using substract and merge commands. After these filtering steps, the number of usable introns per sample varied between a few hundred ( $n=546$, C. elegans, total RNA) to over ten thousand ( $n=13,790, H$. sapiens, nascent-RNA-seq). These large differences resulted from different sequencing depths, sequencing quality (number of usable reads), and from the complexity of the genome (numbers and sizes of introns, number of alternative isoforms, etc.). In order to avoid artifacts due to the different numbers of introns used per sample, we always contrasted the same sets of introns for each comparison of different conditions (e.g. old versus young, treatment versus control). Further note that certain comparisons were not possible for all species, due to variations in the experimental design. For instance, for mouse kidney only a single time point 
after lifespan intervention (dietary restriction DR, age 3 months) was available, which prevented a comparison of old versus young DR mice, but allowed comparison with ad libitum fed mice of the same young age.

\section{Transcriptional elongation speed}

In order to calculate Pol-II speeds we used RNA-seq data obtained from total RNA (65) and nascent RNA $(56,66)$ enrichment. In contrast to the widely used polyA enrichment method (67), which primarily captures mature, spliced mRNAs and is therefore not suitable to estimate Pol-II speeds based on intronic reads, these methods yield sufficient intronic coverage to quantify elongation rates. To analyze the distribution of intronic reads between conditions, we devised a score for each intron. We fitted the read gradient (slope) along each of selected introns (5'->3'; see above for the filtering criteria). Note that the intron gradient is not influenced by exonucleolytic degradation of excised intron lariats (68) and that this measure is only weakly associated with the expression level of the transcript (16) (Fig. S1).

In order to transform slopes to Pol-II elongation speed we used the following formalism. We assume an intron of length $L$ and we assume that at steady state a constant number of polymerases is initiating and the same number of polymerases is terminating at the end of the intron; i.e. we assume that premature termination inside the intron can be ignored. Polymerases are progressing at a common speed of $k[\mathrm{bp} / \mathrm{min}]$. The average time that it takes a polymerase to traverse the whole intron is hence

$$
\Delta t=L / k
$$

Transcription is initiated at a rate of $n$ polymerases per unit time [1/min]. Hence, the number of polymerases $N$ initiating during $\Delta t$ is:

$$
N=\Delta t \times n
$$


The slope $s$ is the number of transcripts after the distance $L$ minus the number of transcripts at the beginning divided by the length of the intron:

$$
s=\frac{0-N}{L}=\frac{-\Delta t \times n}{L}=\frac{-\frac{L}{k} \cdot n}{L}=\frac{-n}{k}
$$

and thus the speed $k$ can be computed from the slope as:

$$
k=\frac{-n}{s}
$$

Hence, slope and speed are inversely related and the speed depends also on the initiation rate (i.e. the expression rate). However, we observed empirically only a small dependency between expression and slope (16) (Fig. S1).

To validate our estimates of Pol-II speeds we compared our data with experimental values estimated via GRO-seq (15) and tiling microarray data (11). There was a significant correlation (GRO-seq: $\mathrm{R}=0.38$, $\mathrm{p}$-value $=4 \mathrm{e}-5$, compared to time point $25-50$ min see Jonkers et al. (15); Tilling array; $\mathrm{R}=0.99$,p-value= $=2.6 \mathrm{e}-16$ and Fig. S14) between our data and experimentally measured transcriptional elongation values. Thus, we used this model in order to convert our intronic slopes into Pol-II speeds in base pair per minute (bp/min). Note that this conversion to bp/min was done solely to convert the slopes to physically interpretable units and it has no influence on any of our statistical analyses. Further, for human cell lines our transcriptional elongation average estimate ranged from 3.0 to $4.3 \mathrm{~kb} / \mathrm{min}$ depending on tissues and stage (proliferating or senescent) a result in the same order of magnitude as previously obtained average speed estimates (69). Finally, we noted that our Pol-II speed estimates for different introns of the same gene were more similar than Pol-II speed estimates for random pairs of introns, implying that gene-specific factors or local chromatin structure influence Pol-II speed (Fig. S15). 


\section{Mismatches detection}

To avoid detection RNA editing or DNA damage we only considered genomic position with only 1 mismatch detected. Since young and old samples were always handled together, we can exclude that consistent differences in the number of mismatches are due to technical biases. The fraction of RNA editing events is generally relatively low and not expected to globally increase with age (70) .

\section{MNase-seq sample preparation}

Mononucleosomal DNA from proliferating and senescent IMR-90 cells (from two donors) were prepared and sequenced on a Illumina HiSeq4000 platform as previously described (71).

\section{MNase-seq analysis}

We used DANPOS (72) with default parameters to calculate the fuzziness (as the average peak width, where 'width' was quantified as the standard deviation around the peak center (72)) and distance between peak summits. Intron and exons annotations were downloaded from UCSC table utilities (64) and filtered as described in Definition of intronic regions.

\section{Figures}

Species silhouette images where downloaded from phylopic.org. Figures were generated using Adobe Illustrator.

\section{Acknowledgements}

RNA-seq data used in this study is available at GEO database under accession numbers: GSE102537 (require token provided with the submission), GSE92486. We acknowledge the Bloomington Drosophila Stock Center for fly stocks and the Max-Planck-Genome-Centre 
bioRxiv preprint doi: https://doi.org/101101/719864; this version posted August 8,2019 . The copyright holder for this preprint (which was not certified by peer review) is the author/funder, who has granted bioRxiv a license to display the preprint in perpetuity. It is made available under aCC-BY-NC-ND 4.0 International license.

Cologne and the Cologne Center for Genomics for library preparation and sequencing. We thanks Martijn Dollé for providing mice used in the aging kidney experiment. We acknowledge funding from the Max Planck Society, Bundesministerium für Bildung und Forschung Grant SyBACol 0315893A-B (to AB, AA and LP) and the European Research Council under the European Union’s Seventh Framework Programme (FP7/2007-2013)/ERC grant agreement number 268739 to LP.

\section{Competing financial interests}

The authors declare no competing financial interests.

\section{Corresponding authors}

Correspondence to: Argyris Papantonis, Adam Antebi, Linda Partridge, Andreas Beyer

\section{Animal Care (Ethical Statement)}

The DR study involving live mice was performed in accordance with the recommendations and guideline of the Federation of the European Laboratory Animal Science Association (FELASA, EU directive 86/609/EEC), with all protocols approved by the Landesamt für Natur, Umwelt und Verbraucherschutz, Nordrhein-Westfalen (LANUV), Germany (reference numbers: 8.87-50.10.37.09.176, 84-02.04.2015.A437, and 84-02.04.2013.A158) and the Netherlands (IACUC in Bilthoven, NIH/NIA 1PO1 AG 17242). The blood samples reference number ethical statement is: 17-362 and the study was registered to the "Deutsches Register Klinischer Studien (DRKS)" and it's identifier is: DRKS00014637. 


\section{References and notes:}

1. J. E. Darnell, Jr., Variety in the level of gene control in eukaryotic cells. Nature 297, 365-371 (1982).

2. C. Vogel, E. M. Marcotte, Insights into the regulation of protein abundance from proteomic and transcriptomic analyses. Nat Rev Genet 13, 227-232 (2012).

3. Y. Liu, A. Beyer, R. Aebersold, On the Dependency of Cellular Protein Levels on mRNA Abundance. Cell 165, 535-550 (2016).

4. D. L. Bentley, Coupling mRNA processing with transcription in time and space. Nature Reviews Genetics 15, 163-175 (2014).

5. T. Saldi, M. A. Cortazar, R. M. Sheridan, D. L. Bentley, Coupling of RNA Polymerase II Transcription Elongation with Pre-mRNA Splicing. J Mol Biol 428, 2623-2635 (2016).

6. J. W. Conaway, R. C. Conaway, Transcription elongation and human disease. Annu Rev Biochem 68, 301-319 (1999).

7. C. Lopez-Otin, M. A. Blasco, L. Partridge, M. Serrano, G. Kroemer, The hallmarks of aging. Cell 153, 1194-1217 (2013).

8. $\quad$ M. Vermulst et al., Transcription errors induce proteotoxic stress and shorten cellular lifespan. Nat Commun 6, 8065 (2015).

9. S. Rangaraju et al., Suppression of transcriptional drift extends $<i>$ C. elegans $</ i>$ lifespan by postponing the onset of mortality. eLife $4 \% \mathrm{U}$ http://elifesciences.org/lookup/doi/10.7554/eLife.08833, (2015).

10. C. P. Martinez-Jimenez et al., Aging increases cell-to-cell transcriptional variability upon immune stimulation. Science 355, 1433-1436 (2017).

11. Y. Wada et al., A wave of nascent transcription on activated human genes. Proc Natl Acad Sci U S A 106, 18357-18361 (2009).

12. A. Ameur et al., Total RNA sequencing reveals nascent transcription and widespread co-transcriptional splicing in the human brain. Nat Struct Mol Biol 18, 1435-1440 (2011).

13. J. Singh, R. A. Padgett, Rates of in situ transcription and splicing in large human genes. Nat Struct Mol Biol 16, 1128-1133 (2009).

14. G. Fuchs et al., 4sUDRB-seq: measuring genomewide transcriptional elongation rates and initiation frequencies within cells. Genome Biol 15, R69 (2014).

15. I. Jonkers, H. Kwak, J. T. Lis, Genome-wide dynamics of Pol II elongation and its interplay with promoter proximal pausing, chromatin, and exons. eLife $3 \% \mathbf{U}$ http://elifesciences.org/lookup/doi/10.7554/eLife.02407, (2014). 
16. A. Veloso et al., Rate of elongation by RNA polymerase II is associated with specific gene features and epigenetic modifications. Genome Research, gr.171405.171113 \%U http://genome.cshlp.org/content/early/172014/171404/171408/gr.171405.171113 (2014).

17. A. Ori et al., Integrated Transcriptome and Proteome Analyses Reveal Organ-Specific Proteome Deterioration in Old Rats. Cell Systems 1, 224-237 http://www.sciencedirect.com/science/article/pii/S2405471215001106 (2015).

18. T. M. Rogalski, A. M. Bullerjahn, D. L. Riddle, Lethal and amanitin-resistance mutations in the Caenorhabditis elegans ama-1 and ama-2 genes. Genetics 120, 409422 (1988).

19. Y. Chen, D. Chafin, D. H. Price, A. L. Greenleaf, Drosophila RNA polymerase II mutants that affect transcription elongation. J Biol Chem 271, 5993-5999 (1996).

20. N. Fong et al., Pre-mRNA splicing is facilitated by an optimal RNA polymerase II elongation rate. Genes Dev 28, 2663-2676 (2014).

21. F. C. Oesterreich et al., Splicing of Nascent RNA Coincides with Intron Exit from RNA Polymerase II. Cell 165, 372-381 (2016).

22. M. de la Mata et al., A slow RNA polymerase II affects alternative splicing in vivo. Mol Cell 12, 525-532 (2003).

23. J. Y. Ip et al., Global impact of RNA polymerase II elongation inhibition on alternative splicing regulation. Genome Research 21, 390-401 http://genome.cshlp.org/content/321/393/390 (2011).

24. U. Braunschweig et al., Widespread intron retention in mammals functionally tunes transcriptomes. Genome Res 24, 1774-1786 (2014).

25. H. Jung et al., Intron retention is a widespread mechanism of tumor-suppressor inactivation. Nat Genet 47, 1242-1248 (2015).

26. V. Aslanzadeh, Y. Huang, G. Sanguinetti, J. D. Beggs, Transcription rate strongly affects splicing fidelity and cotranscriptionality in budding yeast. Genome Res 28, 203-213 (2018).

27. L. Herzel, K. Straube, K. M. Neugebauer, Long-read sequencing of nascent RNA reveals coupling among RNA processing events. Genome Res 28, 1008-1019 (2018).

28. H. L. Drexler, K. Choquet, L. S. Churchman, Human co-transcriptional splicing kinetics and coordination revealed by direct nascent RNA sequencing. bioRxiv, 611020 (2019).

29. P. Mazin et al., Widespread splicing changes in human brain development and aging. Mol Syst Biol 9, 633 (2013).

30. J. R. Tollervey et al., Analysis of alternative splicing associated with aging and neurodegeneration in the human brain. Genome Research 21, 1572-1582 \%U http://genome.cshlp.org/content/1521/1510/1572 (2011). 
31. B. P. Lee et al., Changes in the expression of splicing factor transcripts and variations in alternative splicing are associated with lifespan in mice and humans. Aging Cell 15, 903-913 (2016).

32. C. Heintz et al., Splicing factor 1 modulates dietary restriction and TORC1 pathway longevity in C. elegans. Nature 541, 102-106 (2017).

33. J. K. Pickrell, A. A. Pai, Y. Gilad, J. K. Pritchard, Noisy splicing drives mRNA isoform diversity in human cells. PLoS Genet 6, e1001236 (2010).

34. N. Stepankiw, M. Raghavan, E. A. Fogarty, A. Grimson, J. A. Pleiss, Widespread alternative and aberrant splicing revealed by lariat sequencing. Nucleic Acids Res 43, 8488-8501 (2015).

35. C. Cocquerelle, B. Mascrez, D. Hetuin, B. Bailleul, Mis-splicing yields circular RNA molecules. FASEB J 7, 155-160 (1993).

36. J. M. Nigro et al., Scrambled exons. Cell 64, 607-613 (1991).

37. Y. Zhang et al., The Biogenesis of Nascent Circular RNAs. Cell Rep 15, 611-624 (2016).

38. X.-O. Zhang et al., Complementary Sequence-Mediated Exon Circularization. Cell 159, 134-147 http://www.sciencedirect.com/science/article/pii/S0092867414011118 (2014).

39. K. Grube, A. Burkle, Poly(ADP-ribose) polymerase activity in mononuclear leukocytes of 13 mammalian species correlates with species-specific life span. Proc Natl Acad Sci U S A 89, 11759-11763 (1992).

40. S. Venkatesh, J. L. Workman, Histone exchange, chromatin structure and the regulation of transcription. Nature Reviews Molecular Cell Biology 16, 178-189 (2015).

41. I. Jonkers, J. T. Lis, Getting up to speed with transcription elongation by RNA polymerase II. Nature Reviews Molecular Cell Biology 16, 167-177 (2015).

42. S. Jimeno-González et al., Proceedings of the National Academy of Sciences of the United States of America 112, 14840-14845 (2015).

43. J. Feser et al., Elevated Histone Expression Promotes Life Span Extension. Molecular Cell 39, 724-735

http://www.sciencedirect.com/science/article/pii/S1097276510006258 (2010).

44. Z. Hu et al., Nucleosome loss leads to global transcriptional up-regulation and genomic instability during yeast aging. Genes \& Development 28, 396-408 http://genesdev.cshlp.org/content/328/394/396 (2014).

45. H. Tilgner et al., Nucleosome positioning as a determinant of exon recognition. Nat Struct Mol Biol 16, 996-1001 (2009). 
46. A. L. Hughes, O. J. Rando, Mechanisms underlying nucleosome positioning in vivo. Annu Rev Biophys 43, 41-63 (2014).

47. K. Struhl, E. Segal, Determinants of nucleosome positioning. Nature Structural \& Molecular Biology 20, 267-273 \%* (C) 2013 Nature Publishing Group, a division of Macmillan Publishers Limited. All Rights Reserved. \%U http://www.nature.com/nsmb/journal/v2020/n2013/full/nsmb.2506.html (2013).

48. V. Fitz et al., Nucleosomal arrangement affects single-molecule transcription dynamics. Proc Natl Acad Sci U S A, (2016).

49. T. E. Miller et al., Transcription elongation factors represent in vivo cancer dependencies in glioblastoma. Nature 547, 355-359 (2017).

50. D. A. Bushnell, P. Cramer, R. D. Kornberg, Structural basis of transcription: alphaamanitin-RNA polymerase II cocrystal at 2.8 A resolution. Proc Natl Acad Sci U S A 99, 1218-1222 (2002).

51. E. A. Bowman, D. L. Riddle, W. Kelly, Amino Acid Substitutions in the Caenorhabditis elegans RNA Polymerase II Large Subunit AMA-1/RPB-1 that Result in alpha-Amanitin Resistance and/or Reduced Function. G3 (Bethesda) 1, 411-416 (2011).

52. A. L. Greenleaf, L. M. Borsett, P. F. Jiamachello, D. E. Coulter, Alpha-amanitinresistant D. melanogaster with an altered RNA polymerase II. Cell 18, 613-622 (1979).

53. S. Gronke, D. F. Clarke, S. Broughton, T. D. Andrews, L. Partridge, Molecular evolution and functional characterization of Drosophila insulin-like peptides. PLoS Genet 6, e1000857 (2010).

54. O. Hahn et al., Dietary restriction protects from age-associated DNA methylation and induces epigenetic reprogramming of lipid metabolism. Genome Biol 18, 56 (2017).

55. C. Selman et al., Evidence for lifespan extension and delayed age-related biomarkers in insulin receptor substrate 1 null mice. FASEB J 22, 807-818 (2008).

56. S. Melnik et al., Isolation of the protein and RNA content of active sites of transcription from mammalian cells. Nat Protoc 11, 553-565 (2016).

57. A. M. Bolger, M. Lohse, B. Usadel, Trimmomatic: a flexible trimmer for Illumina sequence data. Bioinformatics 30, 2114-2120 (2014).

58. A. Dobin et al., STAR: ultrafast universal RNA-seq aligner. Bioinformatics 29, 15-21 (2013).

59. M. I. Love, W. Huber, S. Anders, Moderated estimation of fold change and dispersion for RNA-seq data with DESeq2. Genome Biol 15, 550 (2014).

60. J. R. Gerstner et al., Removal of unwanted variation reveals novel patterns of gene expression linked to sleep homeostasis in murine cortex. BMC Genomics 17, 727 (2016). 
61. A. Liberzon et al., The Molecular Signatures Database (MSigDB) hallmark gene set collection. Cell Syst 1, 417-425 (2015).

62. S. Durinck et al., BioMart and Bioconductor: a powerful link between biological databases and microarray data analysis. Bioinformatics 21, 3439-3440 (2005).

63. S. Memczak et al., Circular RNAs are a large class of animal RNAs with regulatory potency. Nature 495, 333-338 (2013).

64. D. Karolchik et al., The UCSC Table Browser data retrieval tool. Nucleic Acids Res 32, D493-496 (2004).

65. P. Cui et al., A comparison between ribo-minus RNA-sequencing and polyA-selected RNA-sequencing. Genomics 96, 259-265 (2010).

66. M. Caudron-Herger, P. R. Cook, K. Rippe, A. Papantonis, Dissecting the nascent human transcriptome by analysing the RNA content of transcription factories. Nucleic Acids Res 43, e95 (2015).

67. A. Mortazavi, B. A. Williams, K. McCue, L. Schaeffer, B. Wold, Mapping and quantifying mammalian transcriptomes by RNA-Seq. Nat Methods 5, 621-628 (2008).

68. J. M. Gray et al., SnapShot-Seq: a method for extracting genome-wide, in vivo mRNA dynamics from a single total RNA sample. PLoS One 9, e89673 (2014).

69. X. Darzacq et al., In vivo dynamics of RNA polymerase II transcription. Nat Struct Mol Biol 14, 796-806 (2007).

70. T. Hwang et al., Dynamic regulation of RNA editing in human brain development and disease. Nat Neurosci 19, 1093-1099 (2016).

71. S. Diermeier et al., TNF $\alpha$ signalling primes chromatin for $N F-\kappa B$ binding and induces rapid and widespread nucleosome repositioning. Genome Biology 15, 536 (2014).

72. K. Chen et al., DANPOS: dynamic analysis of nucleosome position and occupancy by sequencing. Genome Res 23, 341-351 (2013).

73. M. Pertea, D. Kim, G. M. Pertea, J. T. Leek, S. L. Salzberg, Transcript-level expression analysis of RNA-seq experiments with HISAT, StringTie and Ballgown. Nat Protoc 11, 1650-1667 (2016). 


\section{Supplementary material}
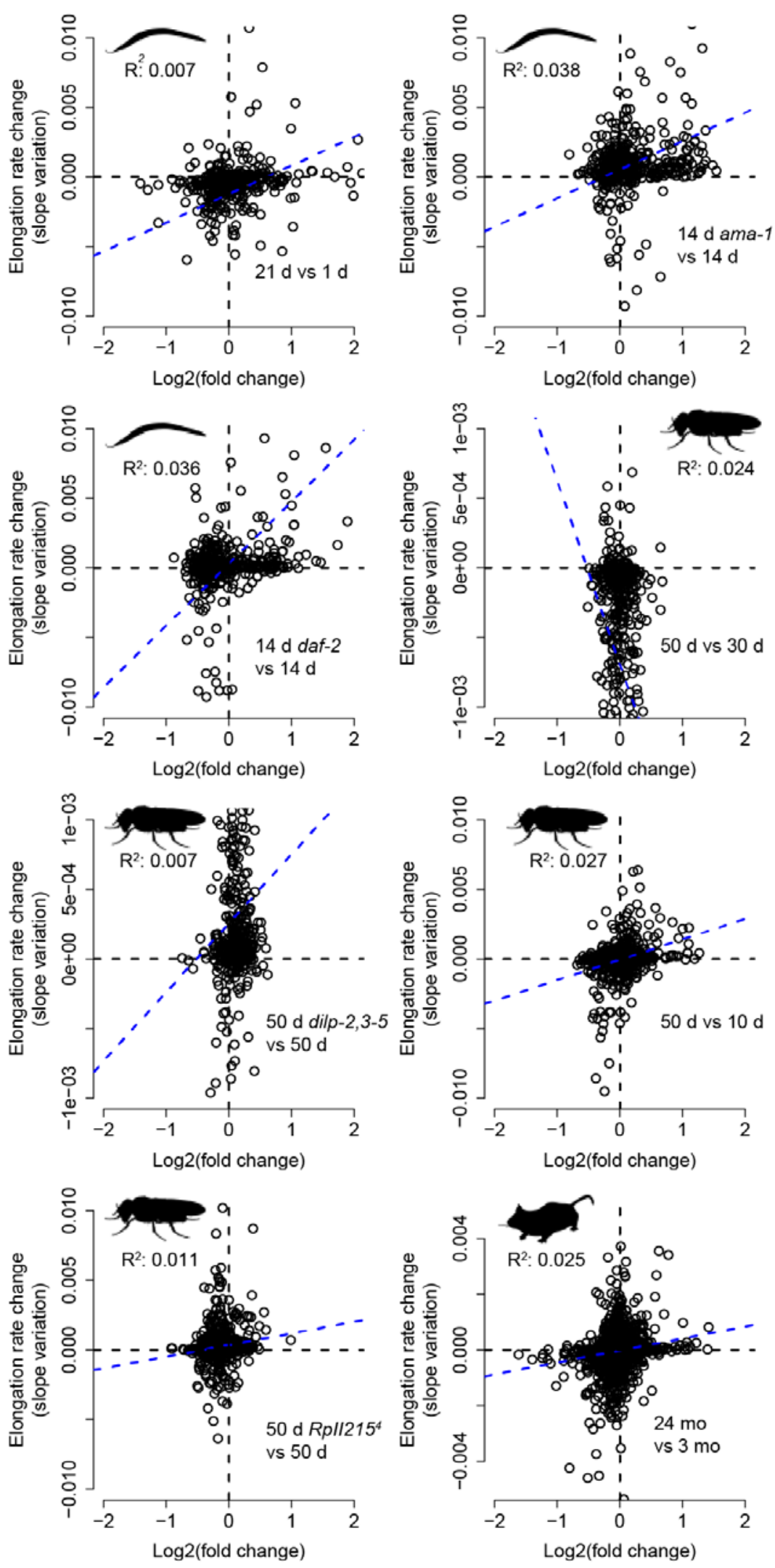

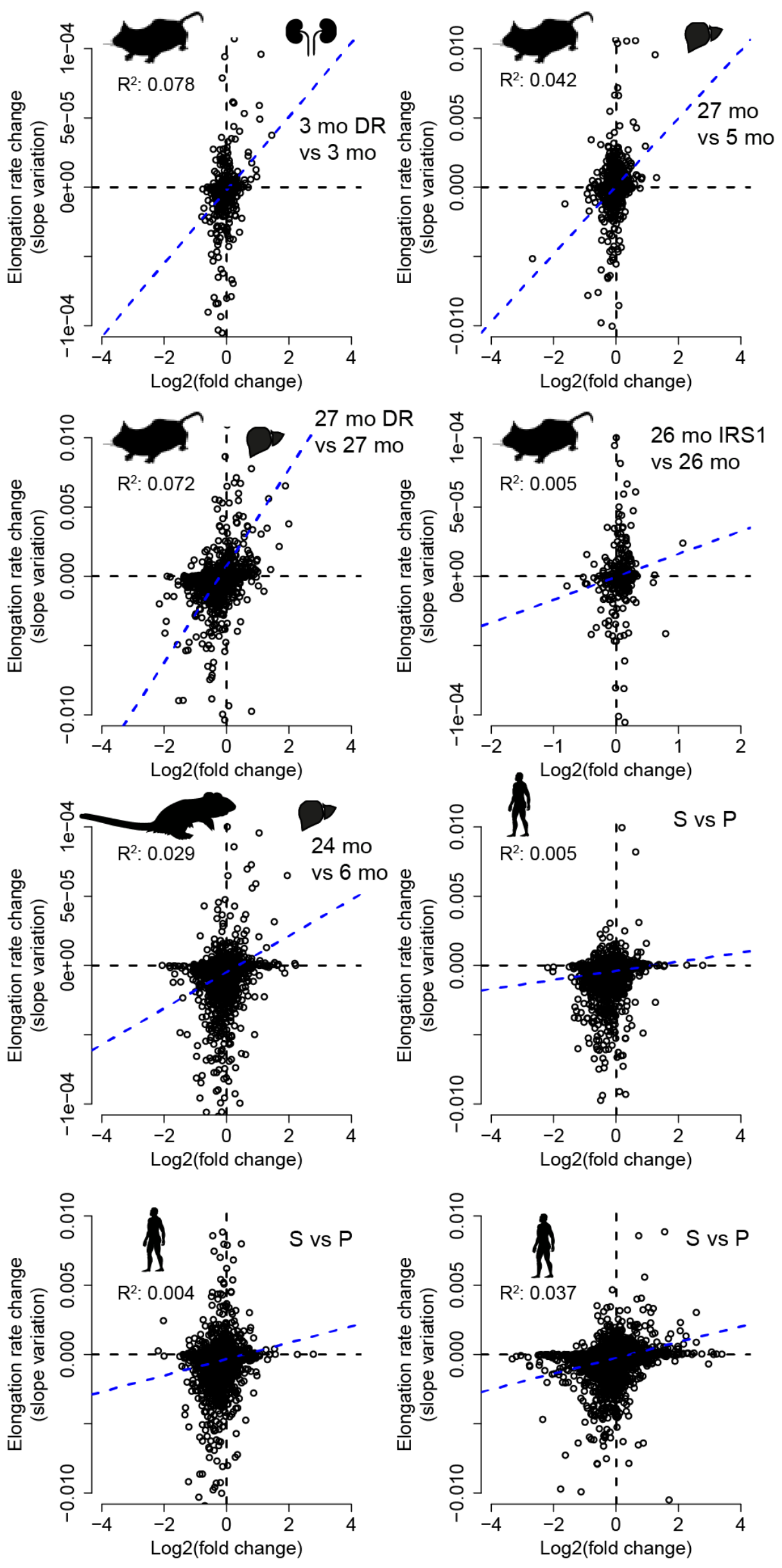
Figure S1 (prev. two pages): Relationship between Pol-II speed and expression levels. Expression levels

( $\log _{2}$ (fold change)) compared to Pol-II speed change for C. elegans (21 d vs $1 \mathrm{~d}$; 14 ama-1(m322) d vs 14 d; 14 daf-2 d vs 14 d), D. melanogaster (heads: 50 d vs 30 d; 50 dilp2,3-5 d vs 50 d; 50 RpII2154 d vs 50 d; 50 d vs 30 d), M. musculus (kidney: 24 mo vs 3 mo; 3 DR mo vs 3 mo), R. norvegicus (liver: 24 mo vs 6 mo), H. sapiens (HUVECs\&IMR90: Senescent vs Proliferating). Between $0.01 \%$ and $8 \%$ of the Pol-II speed change can be explained by mature RNA expression level variation across comparison of our studies. 

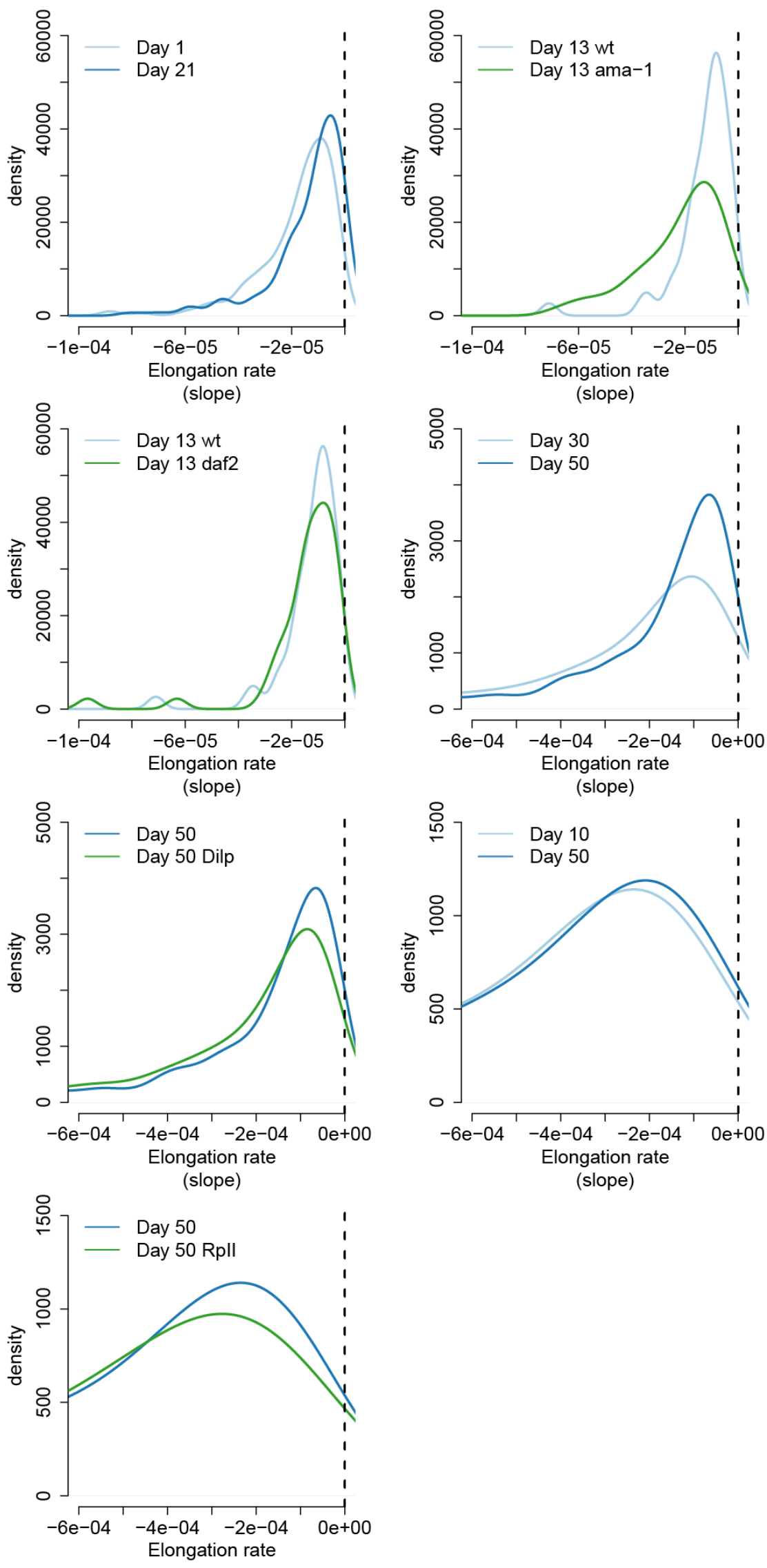

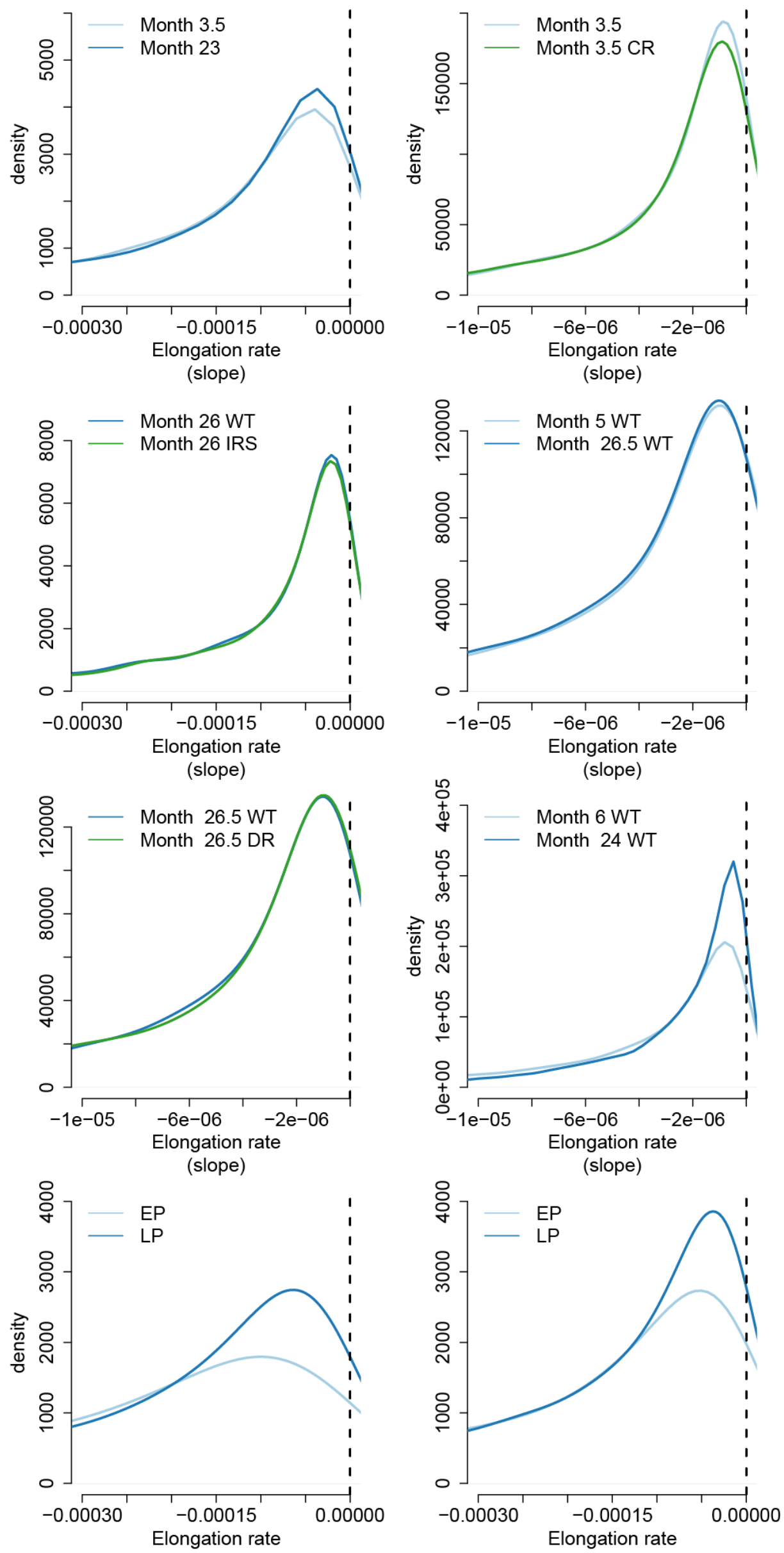
bioRxiv preprint doi: https://doi.org/10.1101/719864; this version posted August 8, 2019. The copyright holder for this preprint (which was

not certified by peer review) is the author/funder, who has granted bioRxiv a license to display the preprint in perpetuity. It is made available under aCC-BY-NC-ND 4.0 International license.

Figure S2 (prev. two pages): Density distribution of intronic slope for each conditions and species (C.

elegans, D. melanogaster, M. musculus, R. norvegicus, H. sapiens (EP: Early passage, LP: Late passage). 
a

$$
\begin{aligned}
& 2.5 \\
& 2 \\
& 1.5 \\
& 1 \\
& 0.5 \\
& 0
\end{aligned}
$$

Significance -Log10(p-value)

b
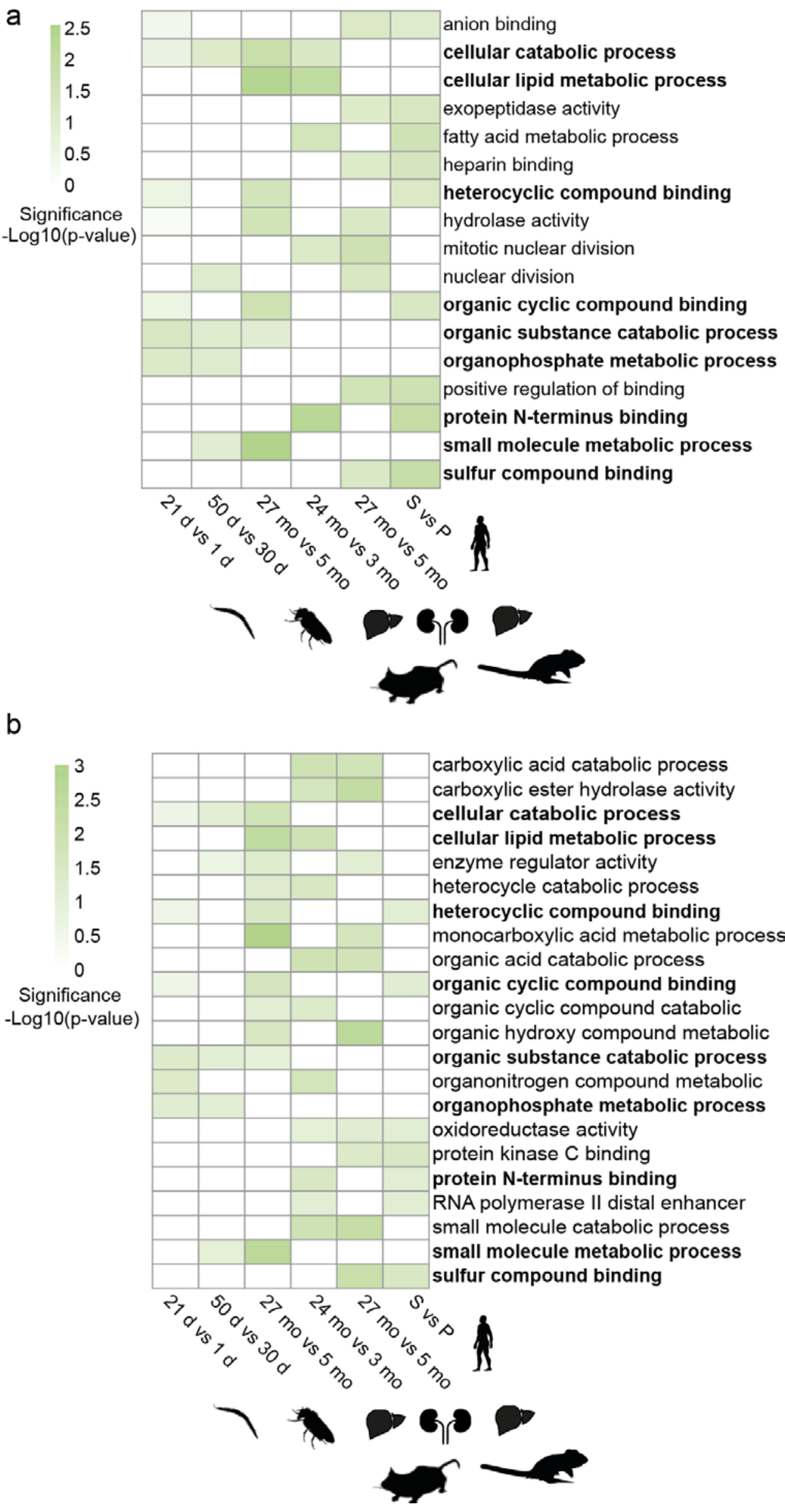


\section{Figure S3 (prev. page): Genes with increase in Pol-II speed are associated with metabolism and}

catabolism related pathway. GO enrichment analysis for genes harboring increasing Pol-II speed change for C. elegans (21 d vs 1 d), D. melanogaster (heads: 50 d vs 30 d), M. musculus (kidney: 24 mo vs 3 mo), $R$. norvegicus (liver: 24 mo vs 6 mo), H. sapiens (IMR90: Senescent vs Proliferating). Using as target set for GO enrichment A, top 200 B, top 300 genes with an increase in Pol-II speed change for each species (common terms between the two set top 200 and top 300 in bold). Color scale represent the significance of enrichment test (all GO term enriched with a p-values under 0.05, with at least more than 10 significant genes for each GO categories, Fisher elim test). 


\section{REGULATION OF DNA TEMPLATED TRANSCRIPTION ELONGATION}

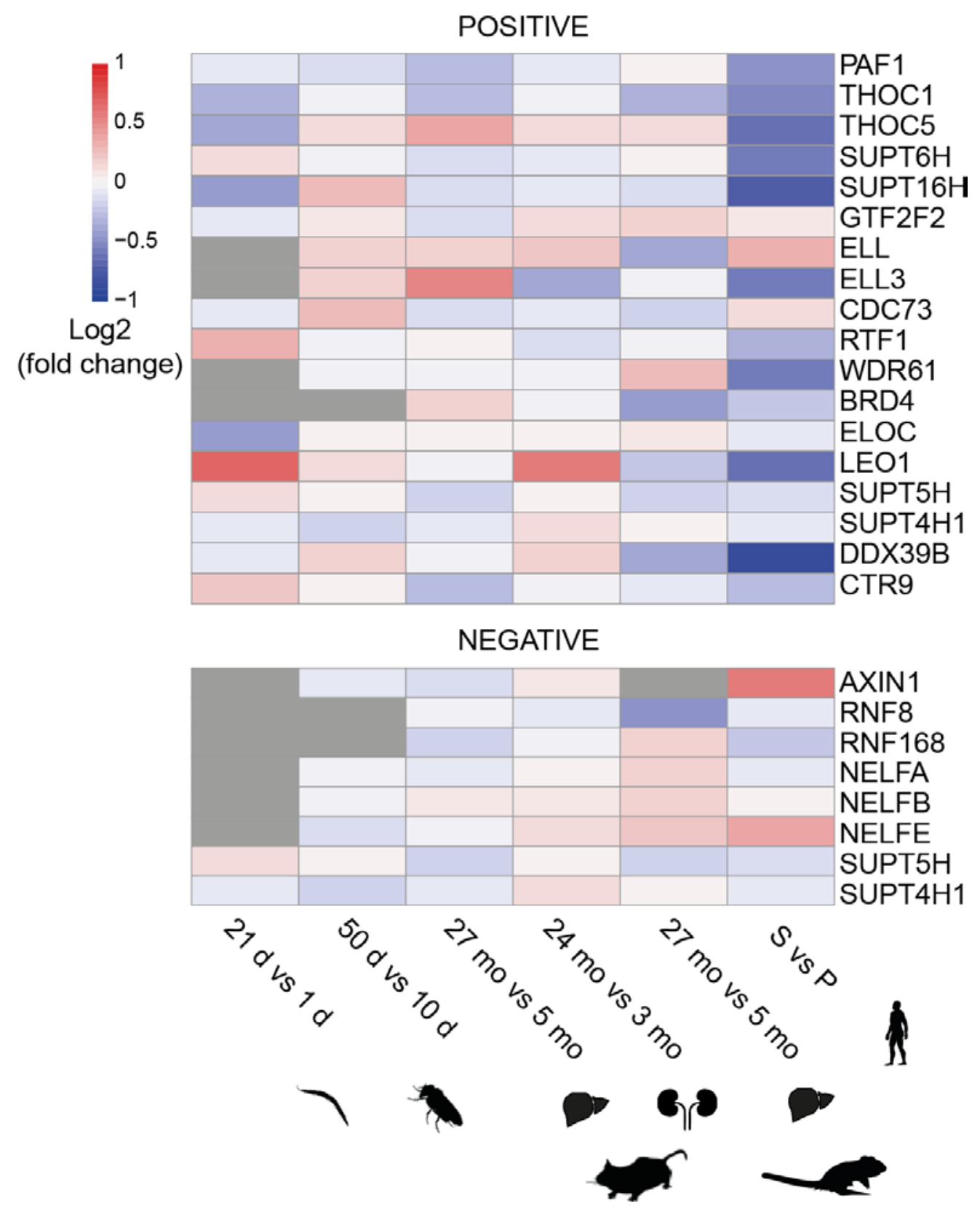

Figure S4: Heat map of differential expression ( $\log _{2}$ fold change) of GSEA/MSigDB (61) annotated genes for 'regulation of DNA templated transcriptional elongation'. Top: activators of transcriptional elongation (POSITIVE); Bottom: repressors of transcriptional elongation (NEGATIVE). Data shown for WT aging time courses: worm (21 d vs 1 d), fly heads (50 d vs 10 d), mouse liver (27 mo vs 5 mo), mouse kidneys (24 mo vs 3 mo) and human fibroblast cell line (IMR90: Senescent vs proliferating). 


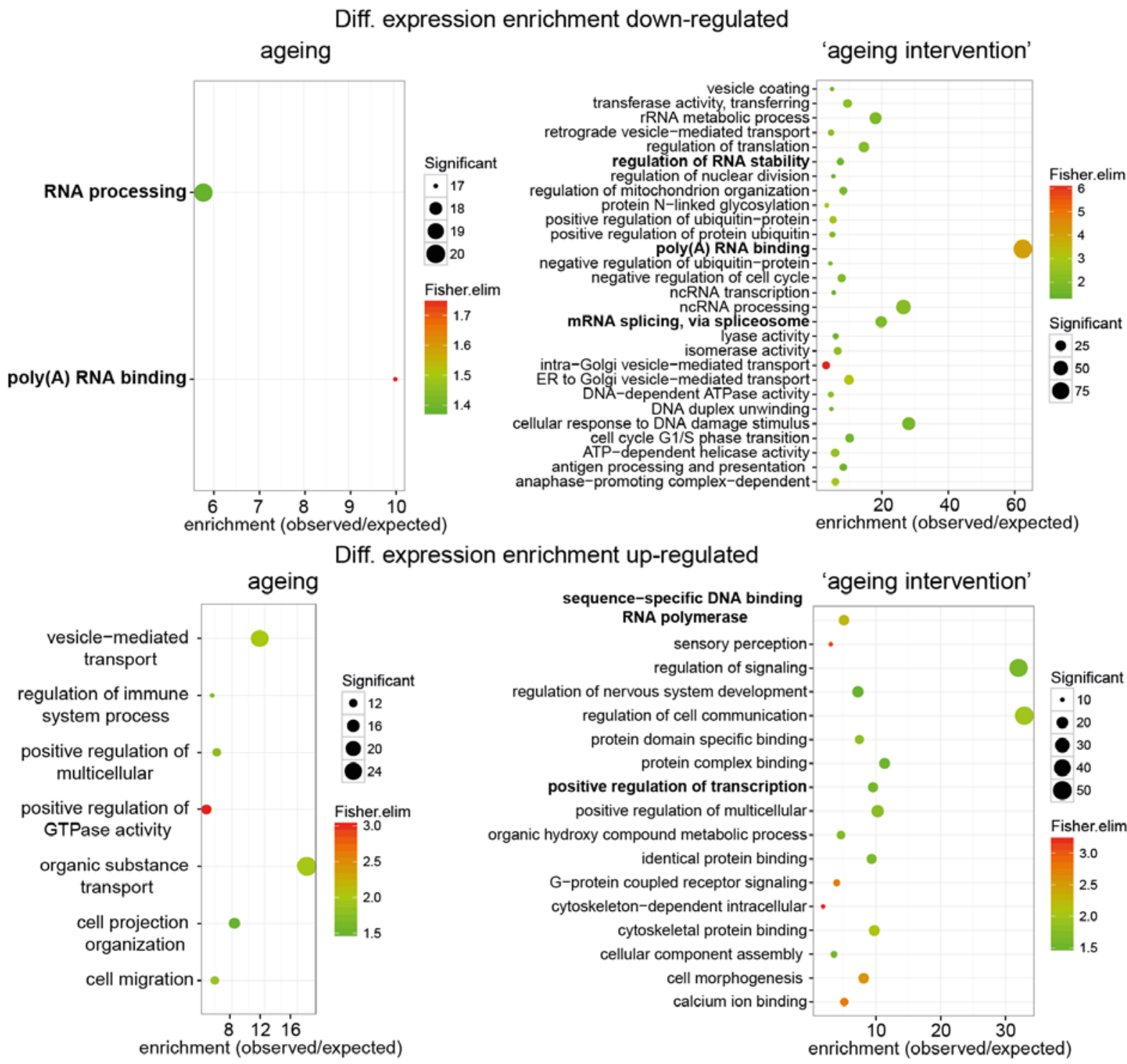

Figure S5: Functional enrichment for across-species differential expression analysis. GO enrichement for consistently up-regulated or down-regulated genes across species during aging or ‘aging intervention’ (as target set: aging up-regulated: 92 genes; aging down-regulated: 71; ‘aging intervention’ up-regulated: 164 genes; ‘aging intervention’ down-regulated: 473 genes; as background set 4784 orthologue genes between $H$. sapiens, R. norvegicus, $M$. musculus, D. melanogaster, C. elegans, all p-values $* \mathrm{P}<0.05$, significant genes $>10$, fisher elim test). GO terms related to transcription and splicing in bold. 
BR1

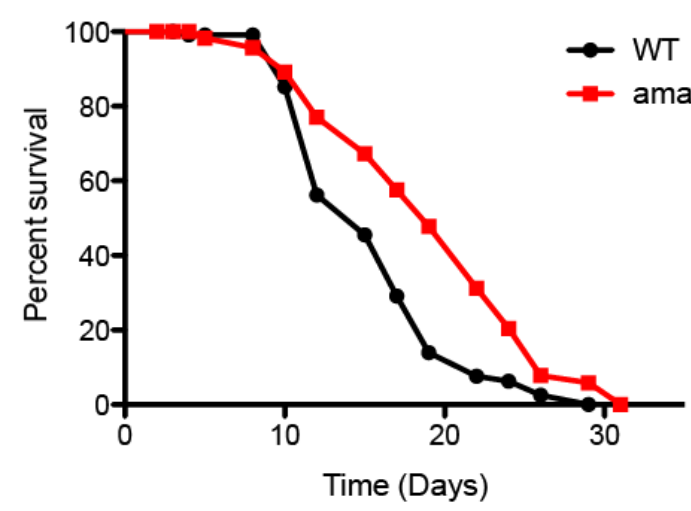

BR3

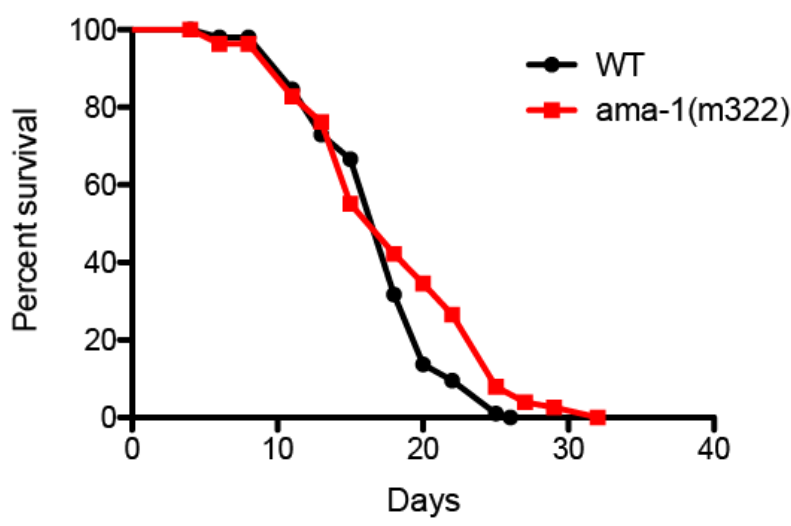

BR2

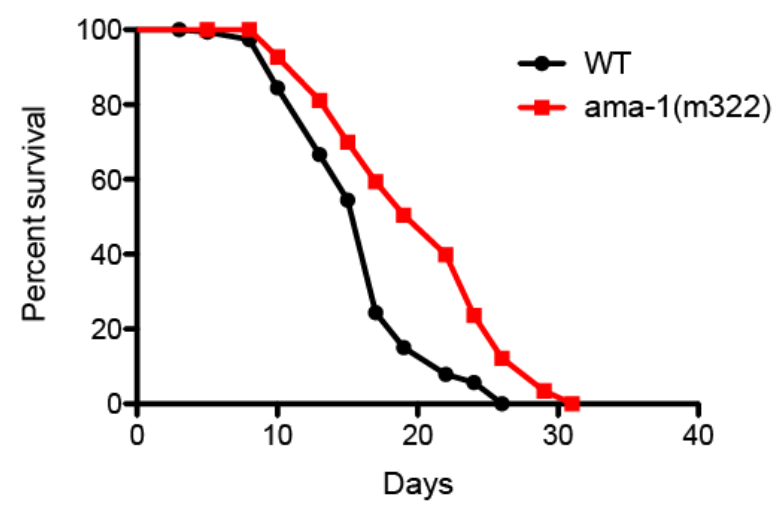

BR4

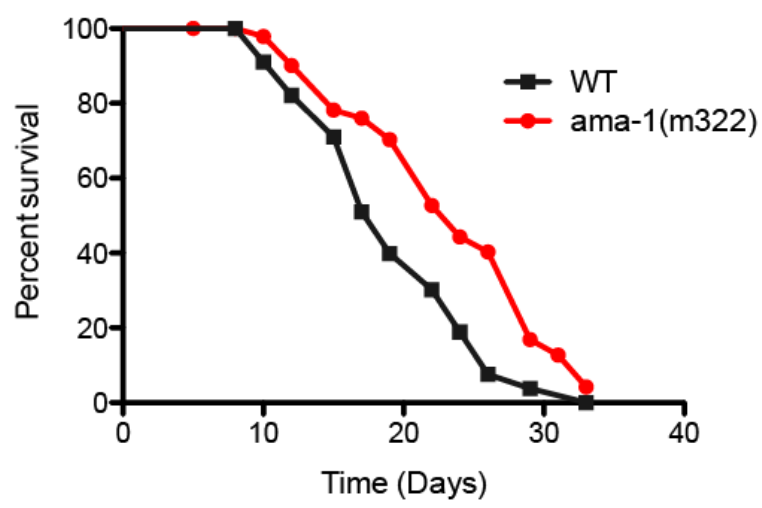

Figure S6: Slowing down Pol-II in C. elegans increases lifespan. Survival of wild-type and ama-1(m322) mutant worms conferring a slow Pol-II elongation rate (4 replicates, BR1:1.267, P < 0,0001; BR2:1.23,P < 0.0001; BR3:1, P = 0.0342; BR4:1.263,P < 0.0001, log-rank test, Mantel-cox). 


\section{pharyngeal pumps/min}

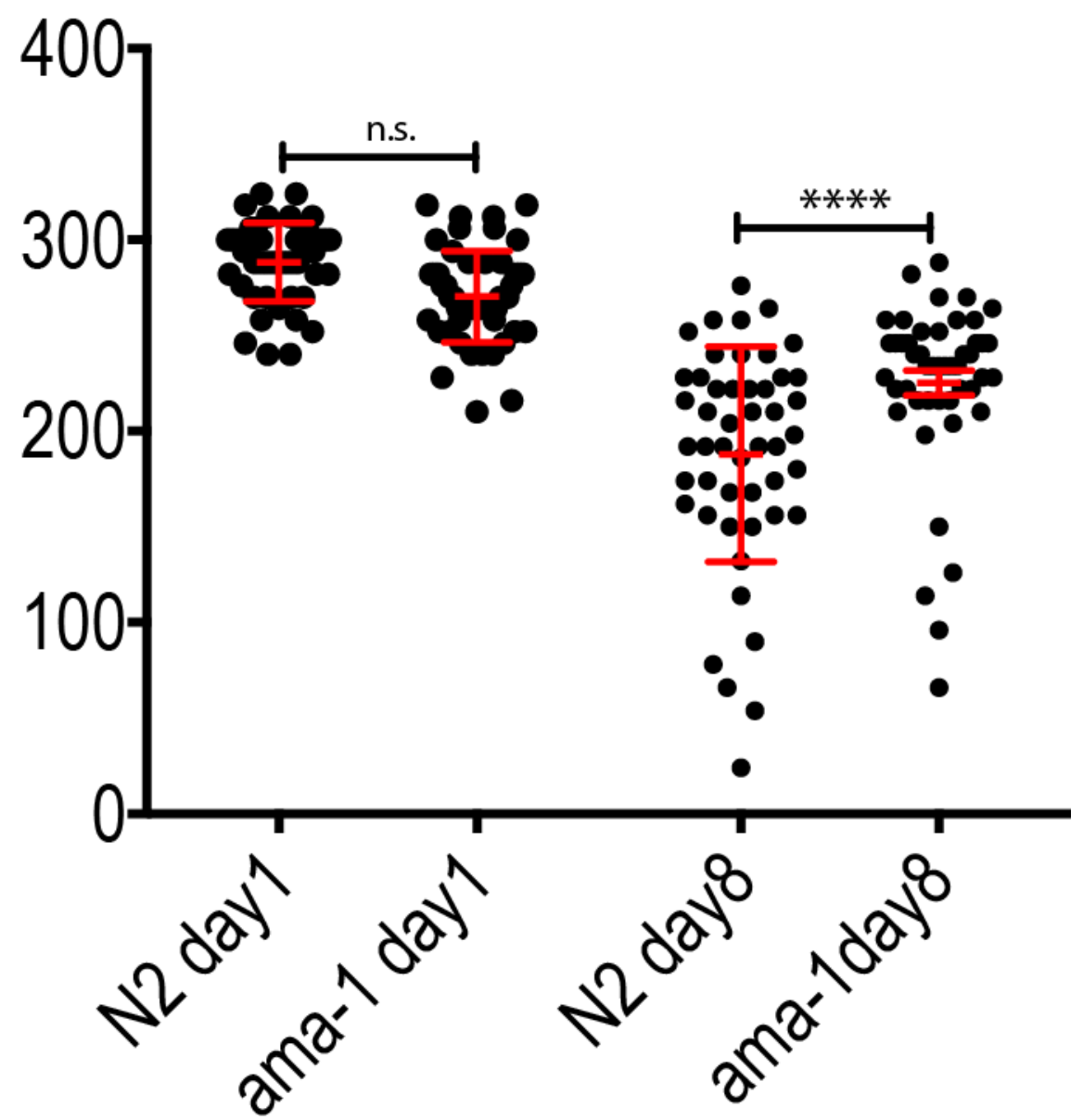

Figure S7: Slowing down Pol-II in C. elegans improves health in old age. The pumping rates of the N2 and ama-1 worms at day 1 and day 8 . There is no difference on day1, but ama-1 worms overall are having much better pumping rates compared to wild types on day8, which correlates with slow aging. Wild types show on the other hand a more heterogeneous pumping rate profile. 


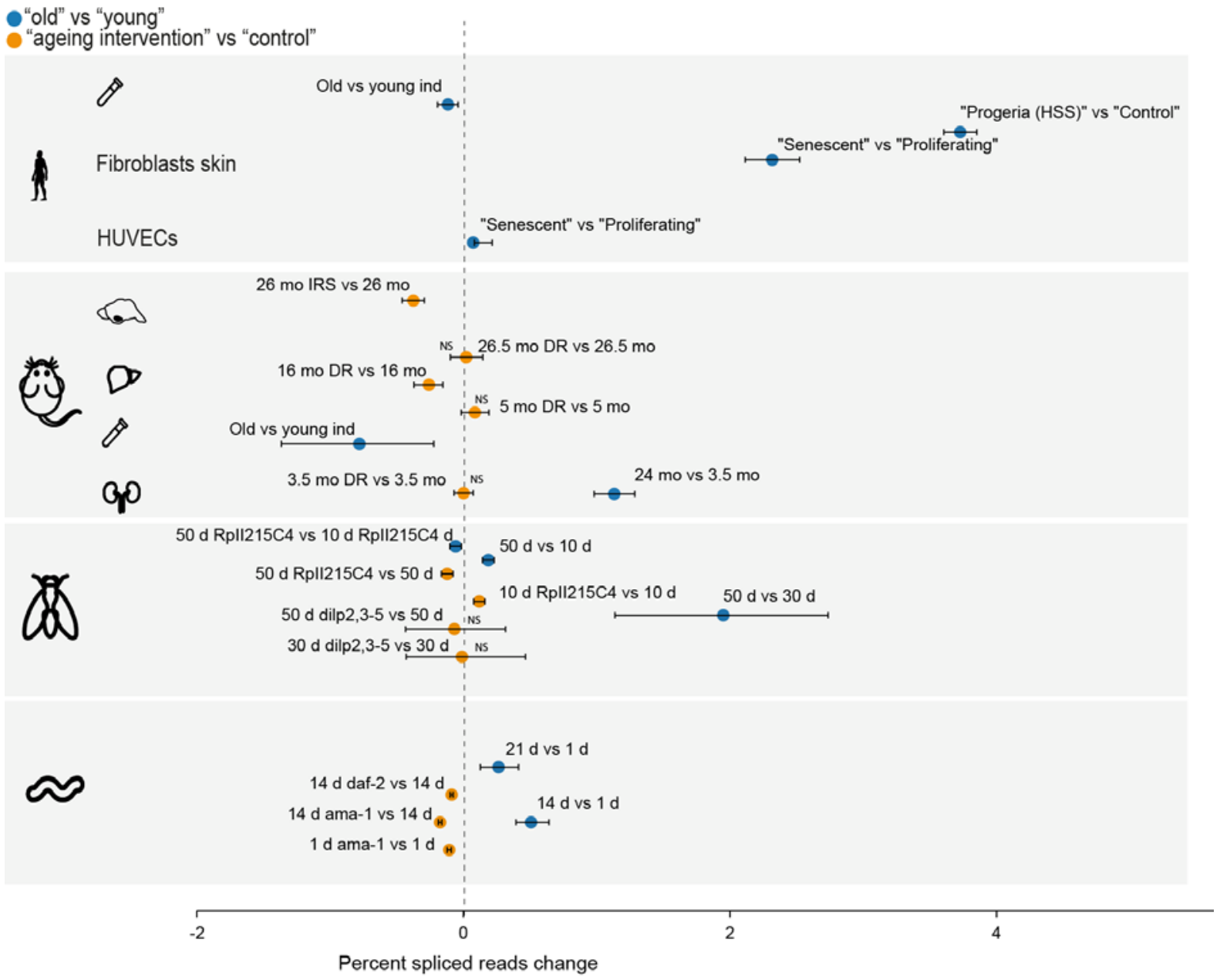

Figure S8: Percent spliced reads increases mostly with age and is often reduced upon slowdown of Pol-II.

Average changes of the fraction of spliced transcripts in worm (whole body), fruit fly (brains), mouse (kidney, liver, hypothalamus), rat (liver) and human cell culture (Fibroblast skin cells derived from a HSS progeria patient compared to a healthy control; HUVECs: umbilical vein endothelial; IMR90: fetal lung). Error bars show median variation $\pm 95 \%$ confidence interval. All average changes of fractions of spliced reads are significantly different from zero ( $\mathrm{P}<0.001$; paired Wilcoxon rank test), except those labeled NS (not significant). Number of genes considered (n) ranged from 124 to 15328. 
a
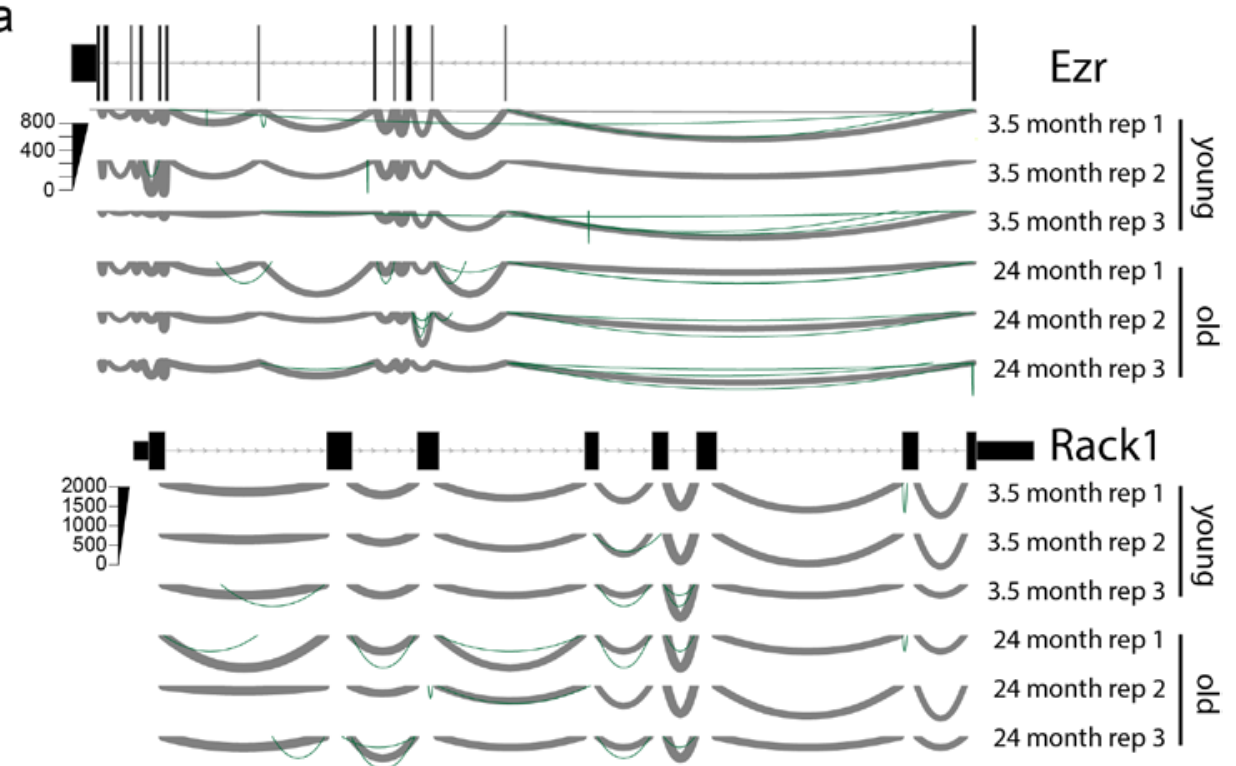

b
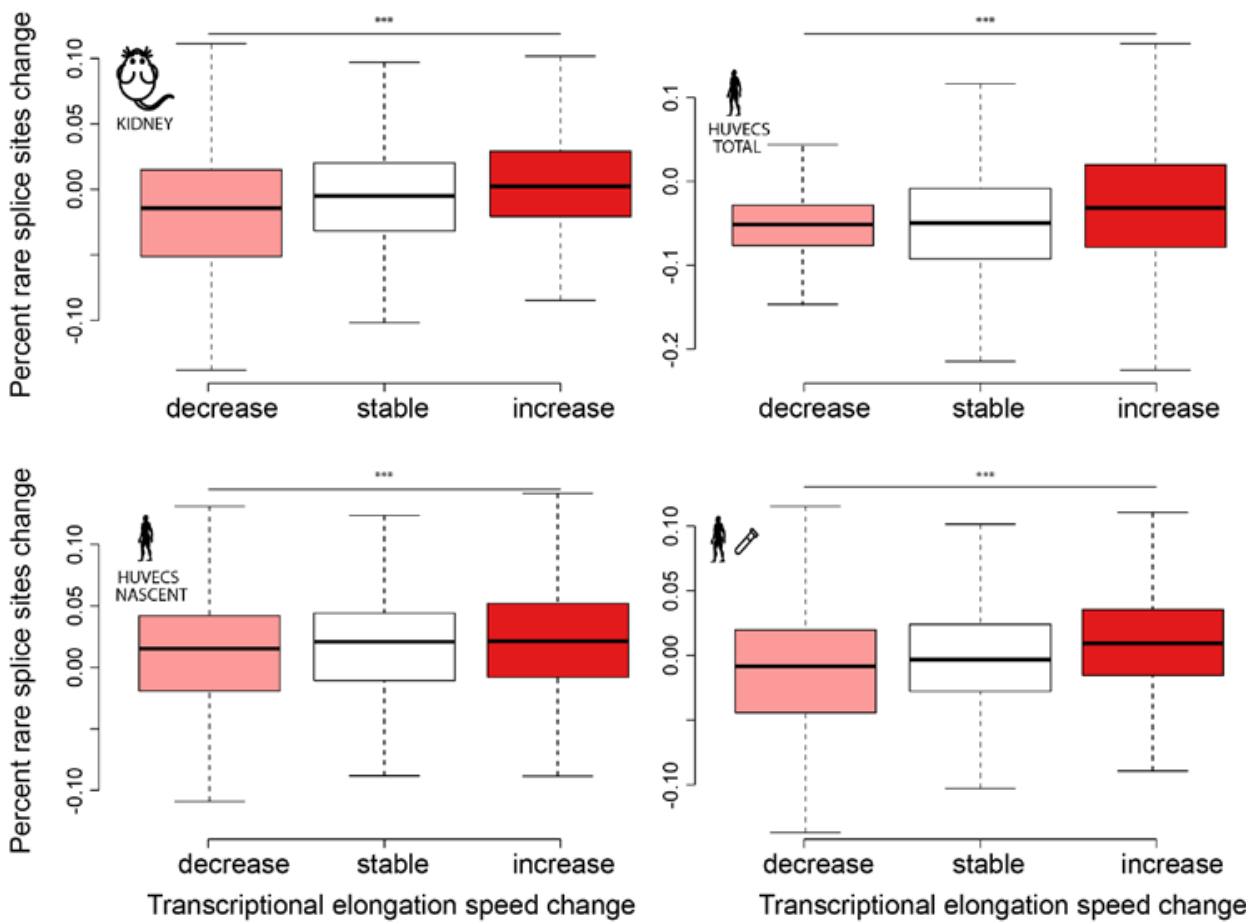

Figure S9: Examples of rare splice sites changes a) for gene Ezr and Rack1 with 3 replicates young (3.5 month) and old (26 month). Line thickness encodes the number of reads supporting this junction. Rare splice sites are shown in green. b) Changes in the fraction of rare splice junctions as a function of Pol-II elongation speed changes.. (Top mouse kidney, human HUVECs total, bottom: human HUVECs nascent, human blood). The differences in mean rare splice site junctions are always significantly different between genes with increased versus decreased elongation speed (***P $<0.001$; paired Wilcoxon rank test). 


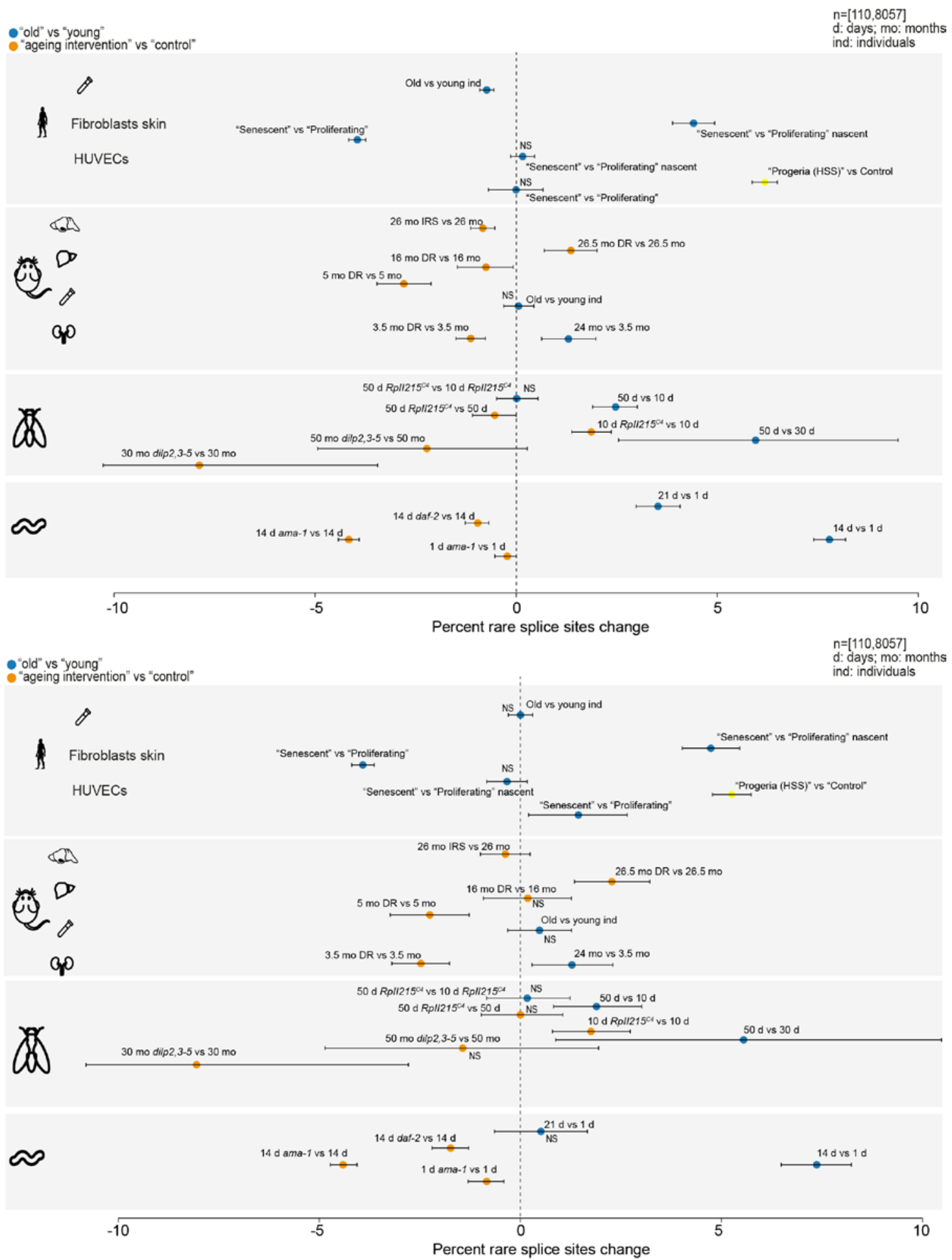

Figure S10: Percent of rare splice events change per gene. Average percent changes of rare splice events (a) $<=0.04$ and $\mathrm{b}$ ) $<=0.1$ percent of total gene expression) with age (blue) and upon lifespan extending treatments (orange). Error bars show median variation $\pm 95 \%$ confidence interval. All changes of average rare splice events are significantly different from zero ( $\mathrm{P}<0.001$; paired Wilcoxon rank test), except those labeled NS (not significant), $* * \mathrm{P}<0.01, \quad *$ : $\mathrm{P}<0.05$. Number of genes considered $(n)$ ranged from 110 to 8057 . 


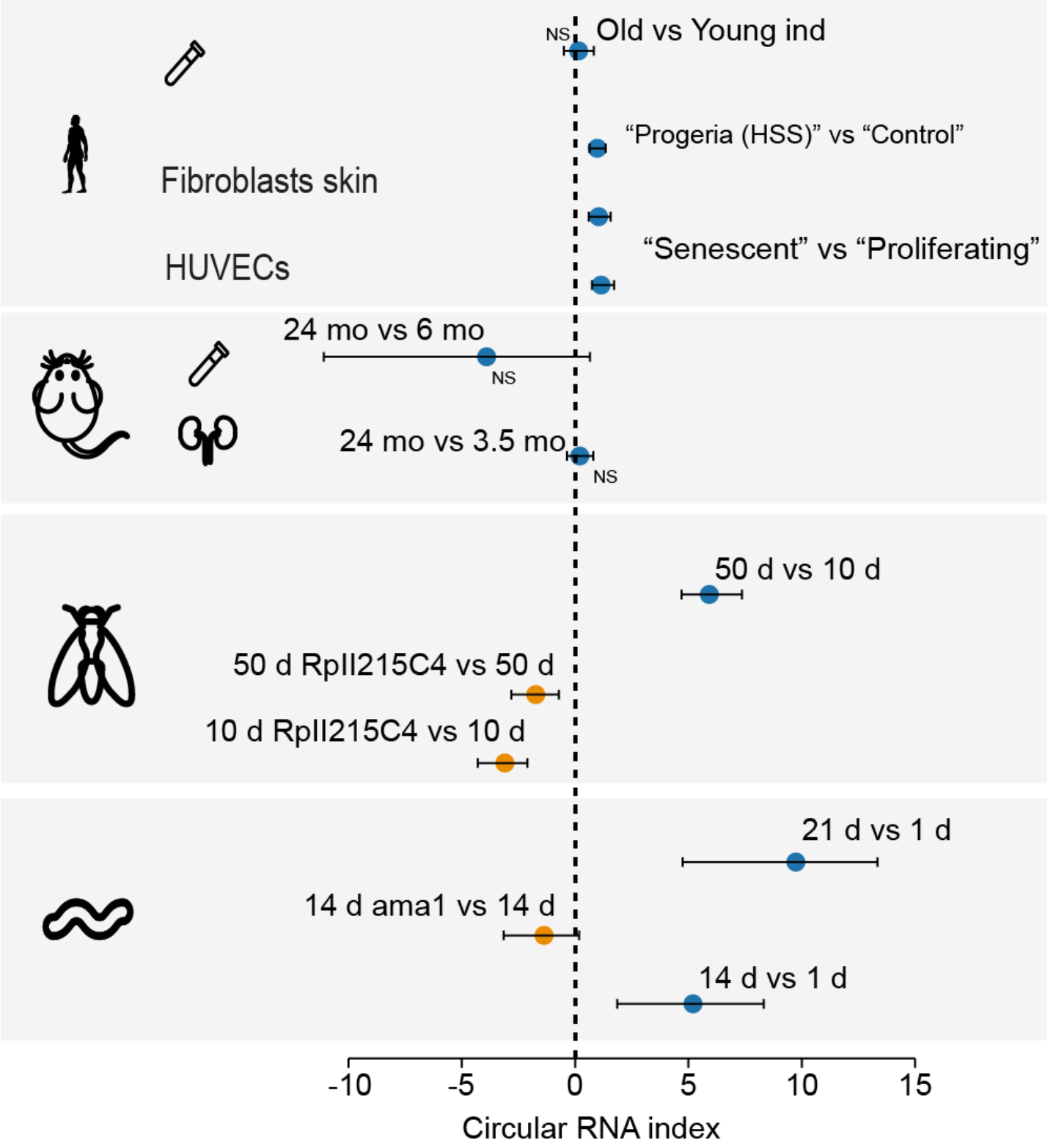

Figure S11: Formation of circular RNAs during aging and under RNA Pol-II slow mutants. Circular RNA index back-splice reads divided by linear splice reads for worms, fly heads, mouse and rat liver, human cell lines. All changes significantly different from zero at $p<0.01$ except those labeled NS: non-significant. Blue for aging or senescence comparison. Orange for RNA Pol-II slow mutants. Yellow for progeria. Error bars show median variation $\pm 95 \%$ confidence interval. 
A
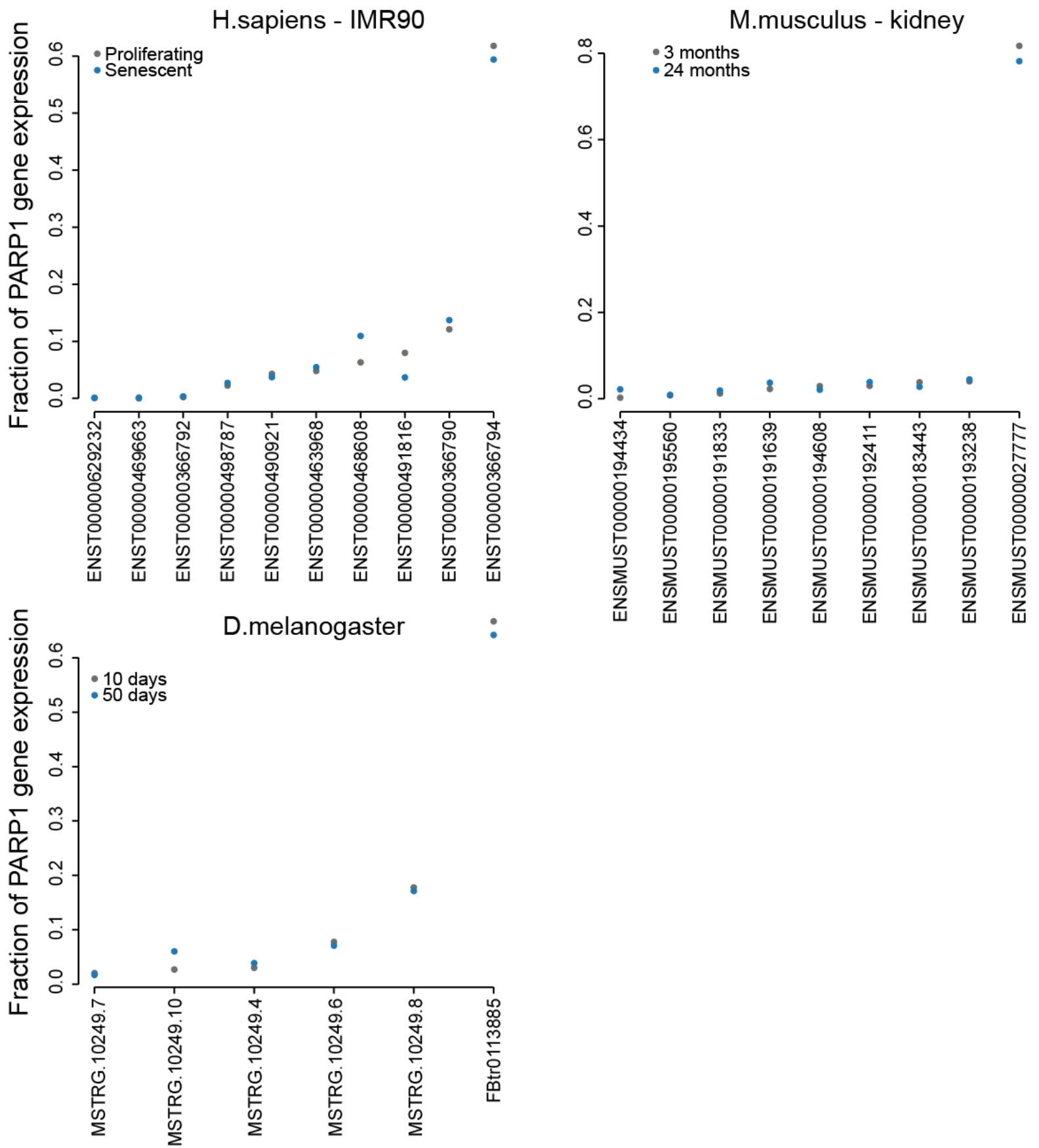

B

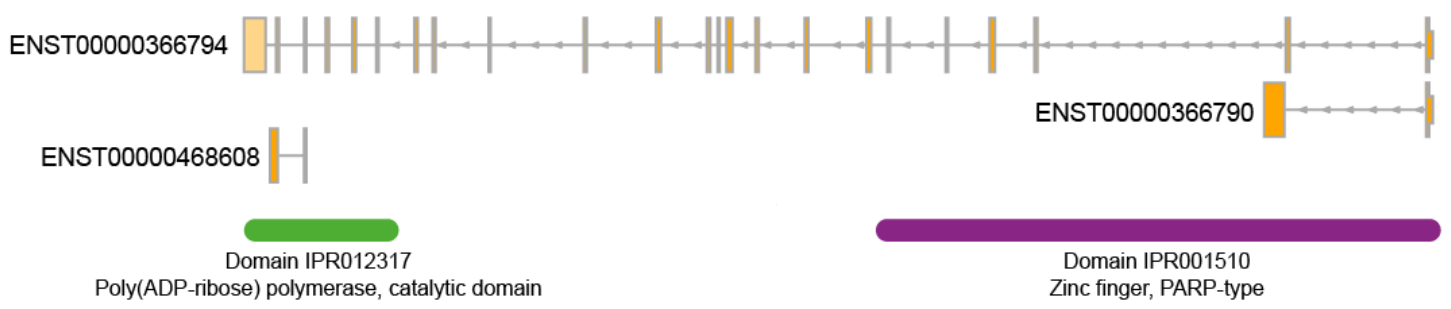




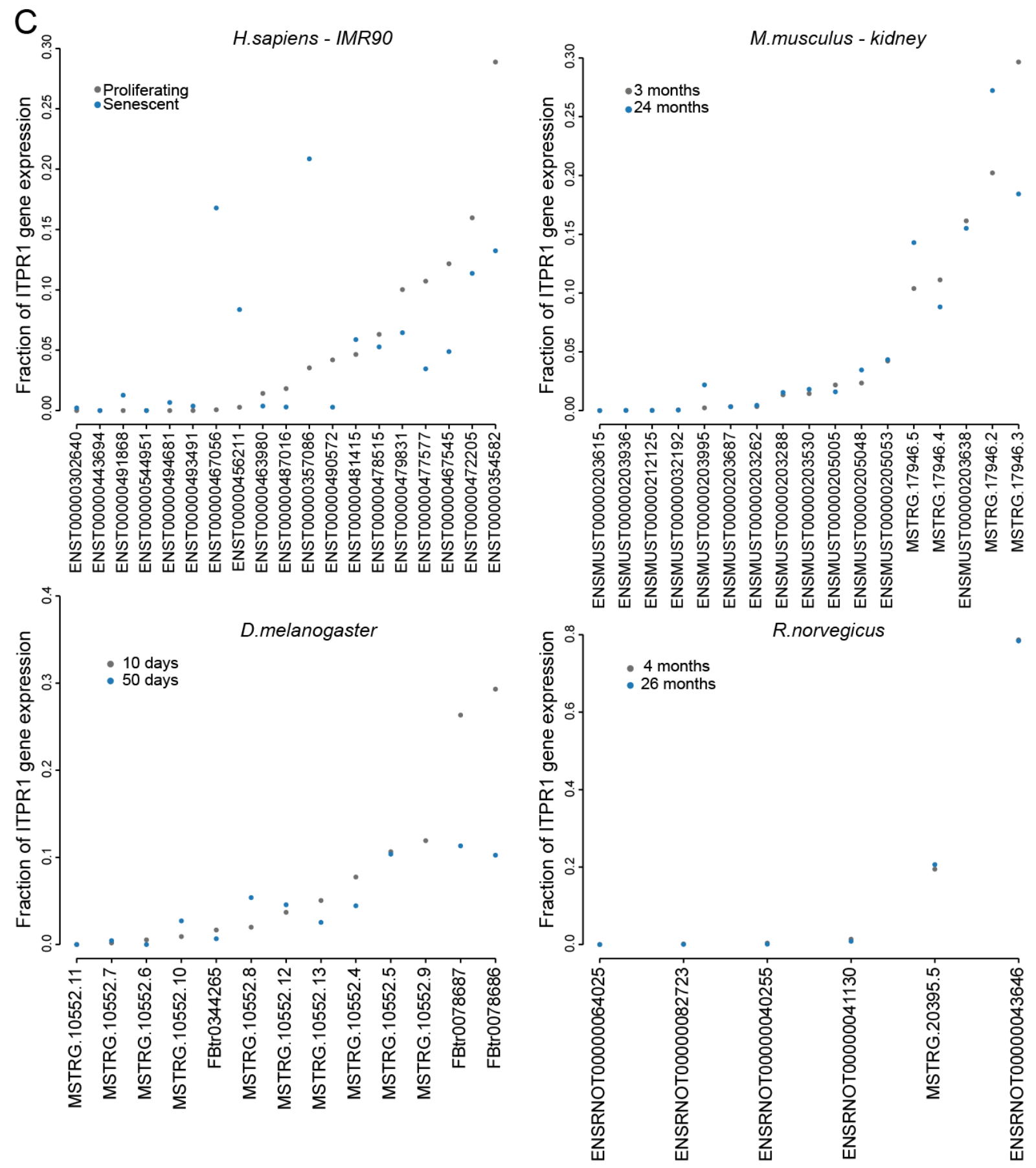

Figure S12: Decline of PARP1 and ITPR1 main isoform and increase in unannotated isoforms in

fibroblast (IMR90) cell lines, mouse kidney, fly brain and rat liver. (A) Dotplots representing the fraction of PARP1 gene expression for each transcripts annotated by ENSEMBL or detected by StringTie (73). Rat data not presented as only one transcript and no new transcript could be identified by StringTie. (B) The alternative isoform represent only a small portion of the main isoform. (C) Dotplots representing the fraction of ITPR1 gene expression for each transcripts annotated by ENSEMBL or detected by StringTie (73). Quantification of transcript abundance was obtain using StringTie. 


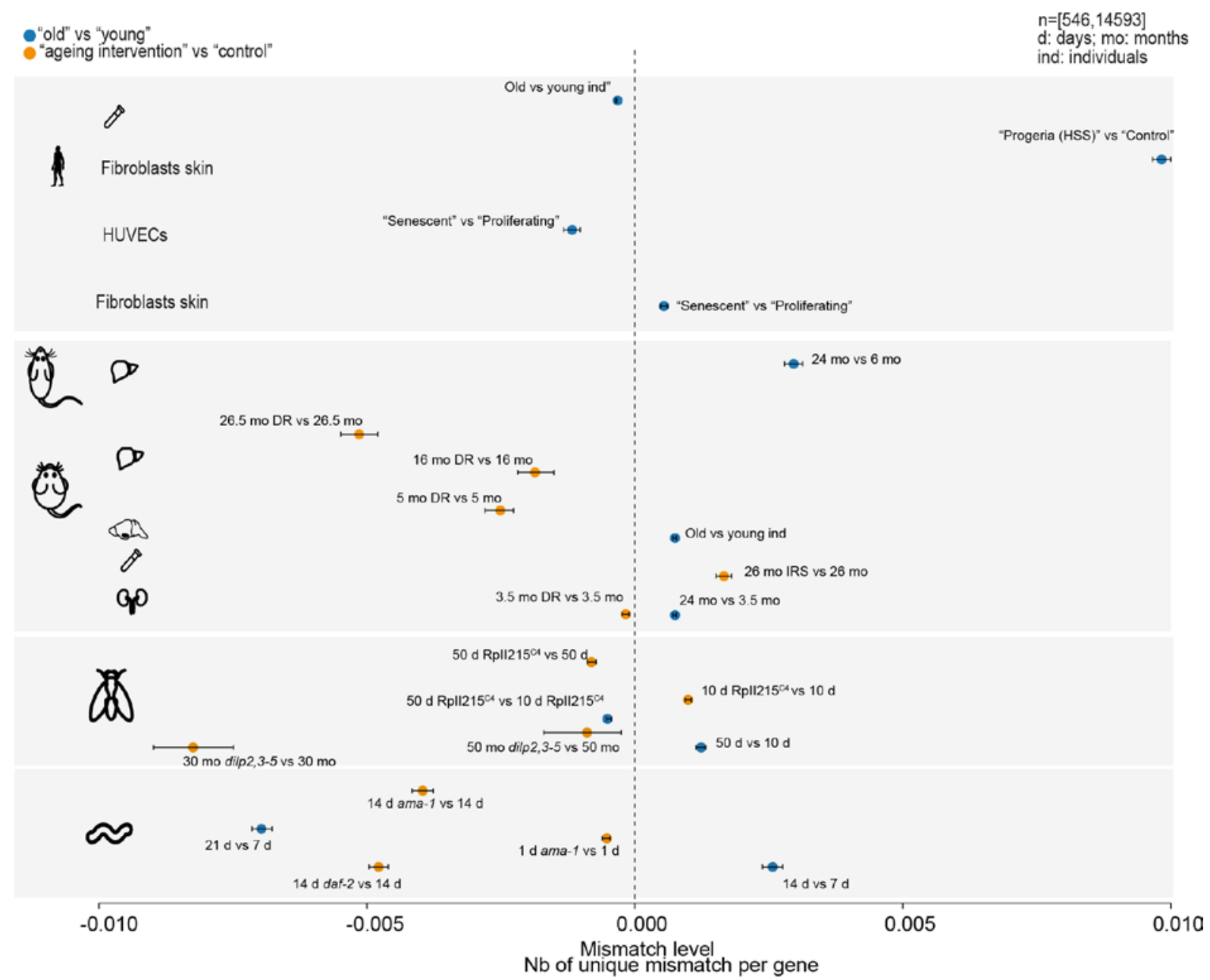

Figure S13: Mismatch level changes during aging and under lifespan-extending interventions. Average mismatch level changes with age (blue) and upon lifespan-extending treatments (orange). Error bars show median variation $\pm 95 \%$ confidence interval. All changes of average mismatches are significantly different from zero (P < 0.05; paired Wilcoxon rank test). Number of genes considered ( $n$ ) ranged from 546 to 14593. 


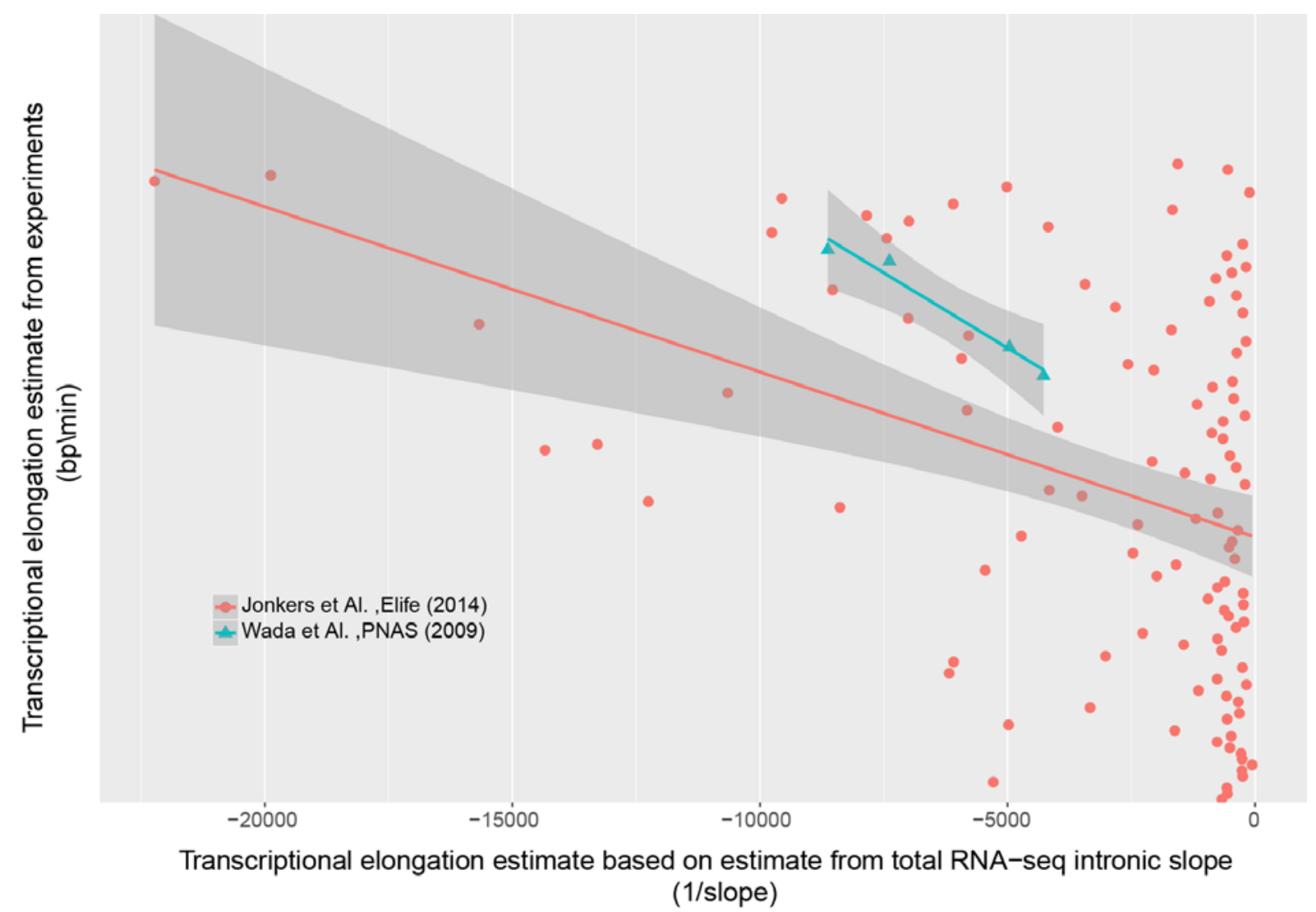

Figure S14: Correlation of transcriptional elongation estimate from GRO-seq (15) and tilling micro-array time series (11) with total RNA intronic gradient. Scatter plot of transcriptional elongation estimate from GRO-seq and tilling microarray time series versus gene-averaged transcriptional elongation estimate from total RNA-seq intron gradient. Each dot represents represent one gene (GRO-seq: Pearson correlation= 0.38, pvalue $=4 \mathrm{e}-05$; Tilling microarray: Pearson correlation $=\mathrm{x}$, p-value $=\mathrm{x}$ ). Red and blue line represent linear fit with 95\% confidence interval for comparison with GRO-seq and tilling array respectively. 

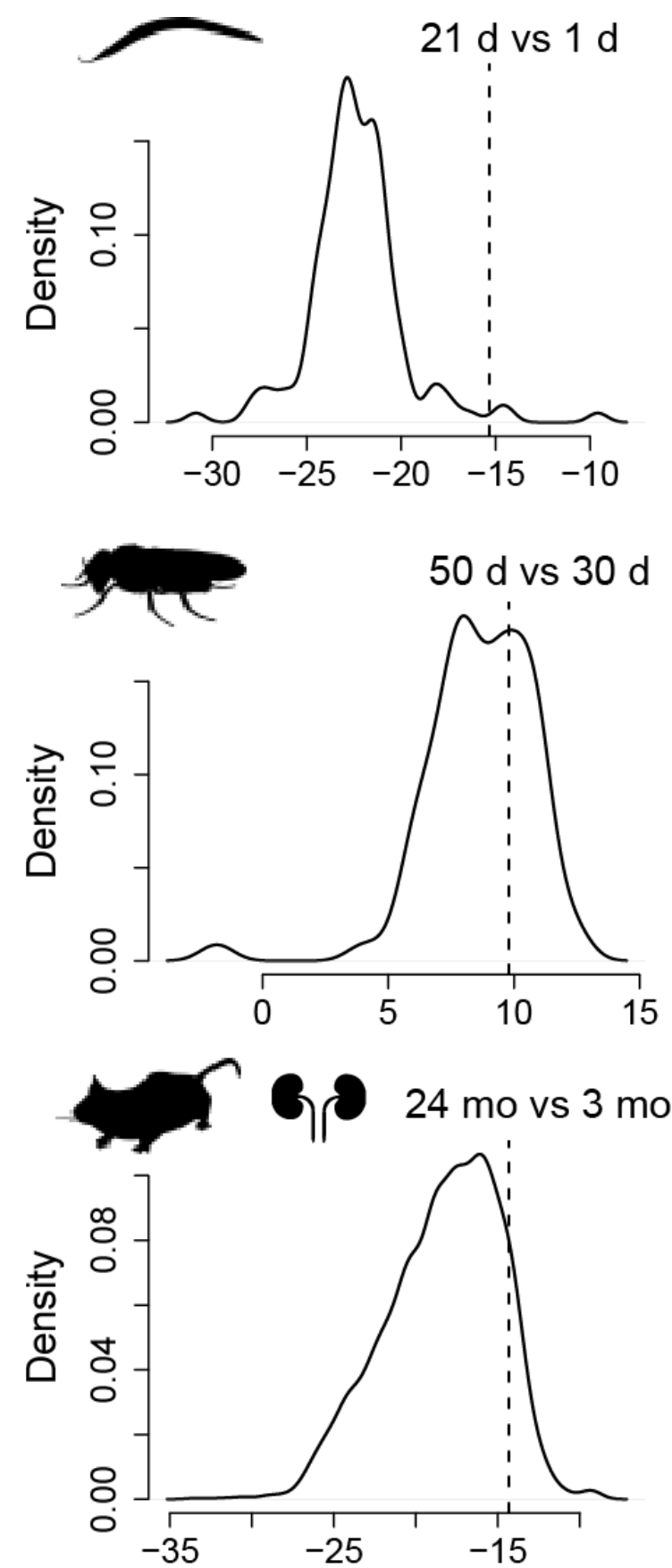

Ca 26 mo IRS1 vs $26 \mathrm{mo}$

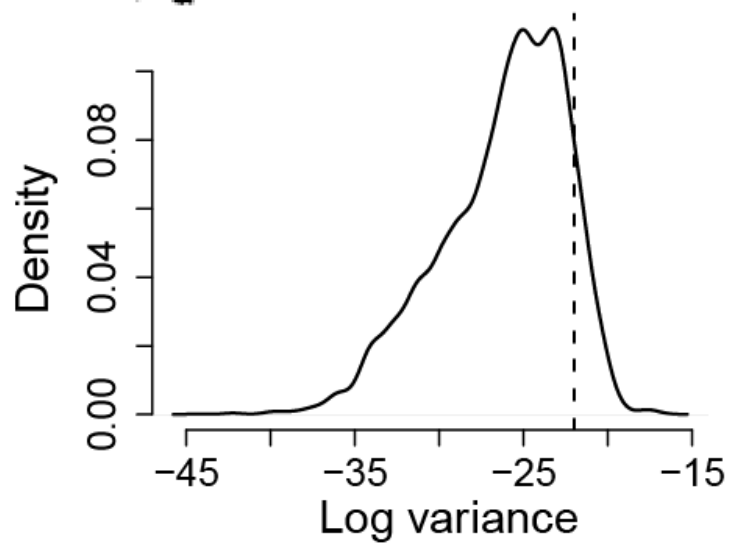

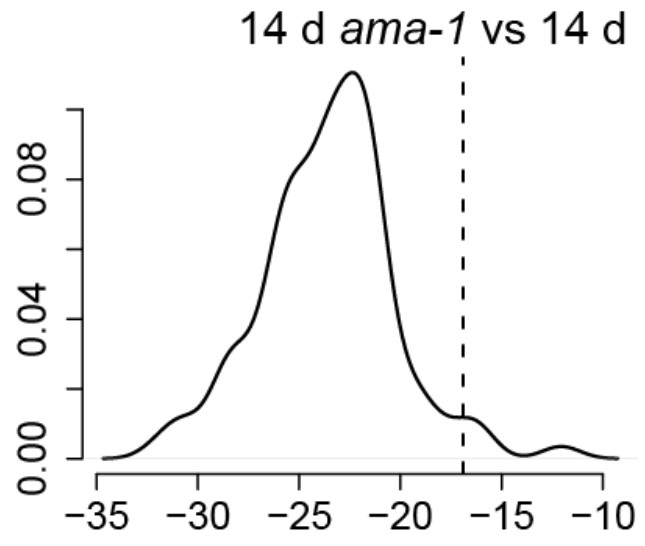
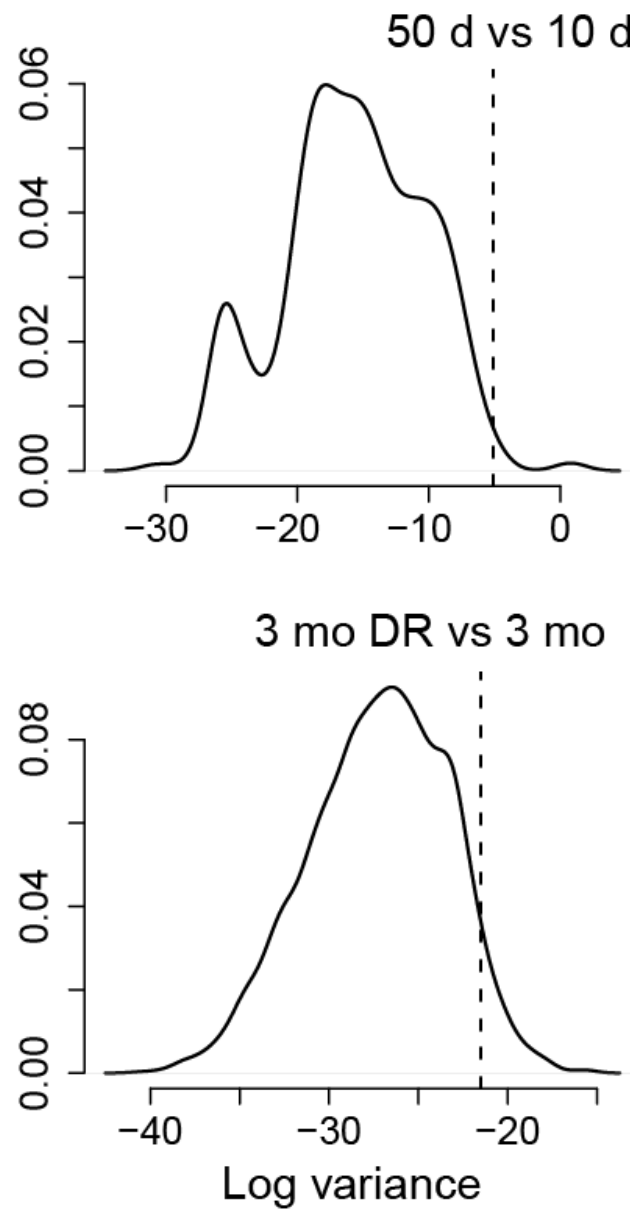

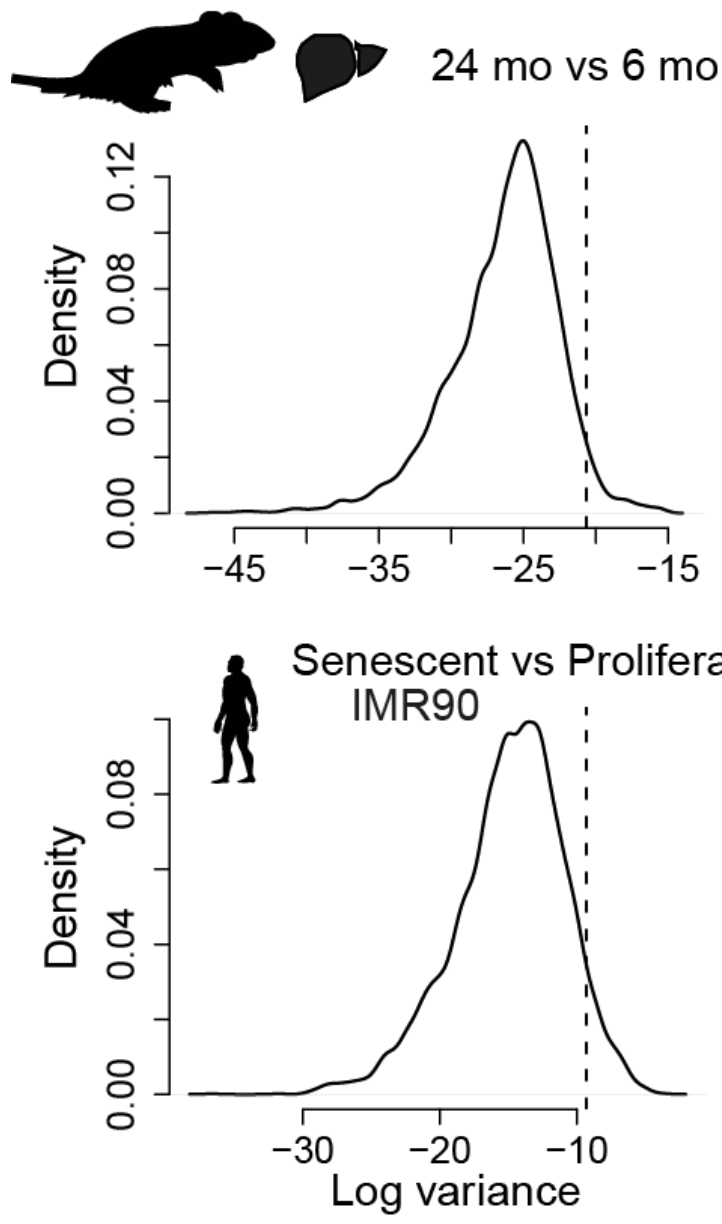

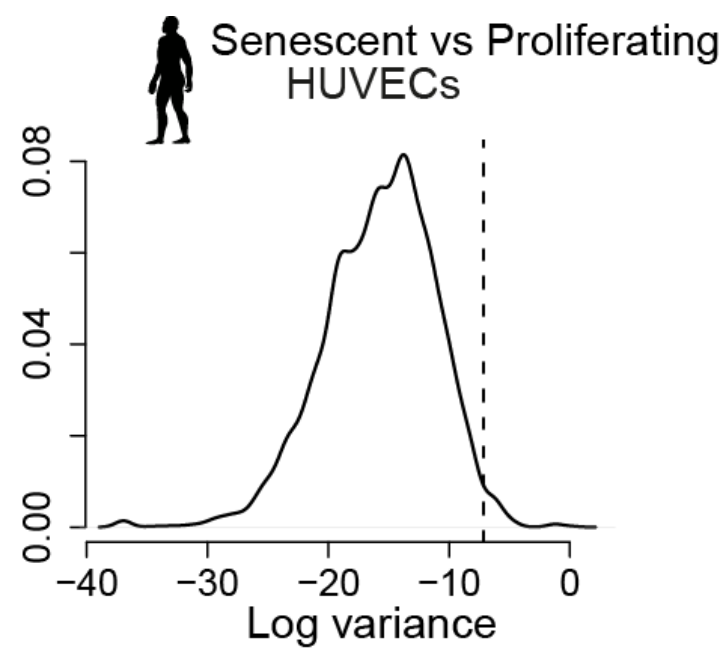

Figure S15 (prev. \& this page): Variation of Pol-II elongation speeds for different introns of the same

gene. Distribution of variances of Pol-II speed estimates (slope per intron) for introns within the same gene.

Average variance of speed estimates across all introns (i.e. between genes; global average) is shown as a dashed vertical line for C. elegans (21 d vs 1 d; 14 daf-2 d vs 14 d), D. melanogaster (heads 50 d vs 30 d; 50 d vs 10 d), M. musculus (kidney: 24 mo vs 3 mo; 3 DR mo vs 3 mo), $R$. norvegicus (liver: 24 mo vs 6 mo), H. sapiens (Umbilical vein endothelial (HUVECs); fibroblast fetal lungs (IMR90): Senescent vs Proliferating). The vast majority of intra-gene variances are below the average inter-gene variance, suggesting that introns of the same gene have coupled Pol-II elongation speeds. 
bioRxiv preprint doi: https://doi.org/101101/719864; this version posted August 8,2019 . The copyright holder for this preprint (which was not certified by peer review) is the author/funder, who has granted bioRxiv a license to display the preprint in perpetuity. It is made available under aCC-BY-NC-ND 4.0 International license.

\begin{tabular}{|c|c|c|c|c|}
\hline Species & Tissue & $\begin{array}{c}\text { Enrichment } \\
\text { protocol }\end{array}$ & $\begin{array}{l}\text { Sequencing parameters } \\
\text { Paired-end/single-end, } \\
\text { read length in base pairs } \\
(b p), \text { millions of reads }(\mathrm{M})\end{array}$ & $\begin{array}{l}\text { Time points }{ }^{1} \text { and } \\
\text { conditions }\end{array}$ \\
\hline \multirow[t]{3}{*}{ C. elegans } & $\begin{array}{l}\text { Whole } \\
\text { body }\end{array}$ & $\begin{array}{l}\text { TruSeq Stranded } \\
\text { Total RNA Library }\end{array}$ & Paired-end, 75 bp, $25 \mathrm{M}$ & $\begin{array}{l}\text { Day } 1(W T) \text {, day } 7(W T) \text {, } \\
\text { day } 14(W T) \text {, day } 21(W T)\end{array}$ \\
\hline & $\begin{array}{l}\text { Whole } \\
\text { body }\end{array}$ & $\begin{array}{c}\text { TruSeq Stranded } \\
\text { Total RNA Library }\end{array}$ & Paired-end, 75 bp, $25 \mathrm{M}$ & $\begin{array}{l}\text { Day } 14(W T, \text { daf- } \\
\text { 2(e1370), ama-1(m322)) }\end{array}$ \\
\hline & $\begin{array}{l}\text { Whole } \\
\text { body }\end{array}$ & $\begin{array}{l}\text { TruSeq Stranded } \\
\text { Total RNA library }\end{array}$ & Paired-end, 75 bp, $25 \mathrm{M}$ & $\begin{array}{l}\text { Day } 1 \text { (WT, ama-1 } \\
(\mathrm{m} 322))\end{array}$ \\
\hline \multirow{2}{*}{$\begin{array}{c}D . \\
\text { melanogaste } \\
r\end{array}$} & Head & $\begin{array}{c}\text { TruSeq Stranded } \\
\text { Total RNA Library }\end{array}$ & $\begin{array}{l}\text { Single-end, } 100 \mathrm{bp}, 37.5 \\
\mathrm{M}\end{array}$ & $\begin{array}{l}\text { Day } 30 \text { (WT, dilp2,3-5), } \\
\text { Day } 50 \text { (WT, dilp2,3-5) }\end{array}$ \\
\hline & Head & $\begin{array}{c}\text { TruSeq Stranded } \\
\text { Total RNA Library }\end{array}$ & Paired-end, $75 b p, 30 \mathrm{M}$ & $\begin{array}{l}\text { Day } 10\left(W T, R p I I 215^{C 4}\right) \text {, } \\
\text { day } 50\left(W T, R p I I 215^{C 4}\right)\end{array}$ \\
\hline \multirow[t]{7}{*}{ M. musculus } & Kidney & $\begin{array}{c}\text { TruSeq Stranded } \\
\text { Total RNA Library }\end{array}$ & Paired-end, 75 bp, $70 \mathrm{M}$ & $\begin{array}{l}\text { Month } 3(W T), \text { month } 24 \\
(W T)\end{array}$ \\
\hline & Kidney & $\begin{array}{l}\text { TruSeq Stranded } \\
\text { Total RNA Library }\end{array}$ & Paired-end, 75 bp, $30 \mathrm{M}$ & $\begin{array}{l}\text { Month } 3(W T, D R) \\
\text { (4 replicates) }\end{array}$ \\
\hline & Liver & $\begin{array}{c}\text { TruSeq Stranded } \\
\text { Total RNA Library }\end{array}$ & Paired-end, 75 bp, $37.5 \mathrm{M}$ & Month $5(W T, D R)$ \\
\hline & Liver & $\begin{array}{l}\text { TruSeq Stranded } \\
\text { Total RNA Library }\end{array}$ & Paired-end, 75 bp, $37.5 \mathrm{M}$ & Month $16(W T, D R)$ \\
\hline & Liver & $\begin{array}{c}\text { TruSeq Stranded } \\
\text { Total RNA Library }\end{array}$ & Paired-end, 75 bp, $37.5 \mathrm{M}$ & Month $27(W T, D R)$ \\
\hline & Blood & $\begin{array}{c}\text { TruSeq Stranded } \\
\text { Total RNA Library }\end{array}$ & Paired-end, 75 bp, $70 \mathrm{M}$ & $\begin{array}{l}\text { Month } 5 \text { (WT), month } 27 \\
(W T)\end{array}$ \\
\hline & $\begin{array}{l}\text { Hypothala- } \\
\text { mus }\end{array}$ & $\begin{array}{c}\text { TruSeq Stranded } \\
\text { Total RNA Library }\end{array}$ & Single-end, $100 \mathrm{bp}, 30 \mathrm{M}$ & Month 26 (WT, IRS1-/-) \\
\hline \multirow{2}{*}{$\begin{array}{c}R . \\
\text { norvegicus } \\
(17)\end{array}$} & Liver & $\begin{array}{c}\text { TruSeq Stranded } \\
\text { Total RNA Library }\end{array}$ & Single-end, $50 \mathrm{bp}, 60 \mathrm{M}$ & $\begin{array}{l}\text { Month } 6(W T), \text { month } 24 \\
(W T) \text { (2 replicates) }\end{array}$ \\
\hline & Brain $^{2}$ & $\begin{array}{c}\text { TruSeq Stranded } \\
\text { Total RNA Library }\end{array}$ & Single-end, $50 \mathrm{bp}, 20 \mathrm{M}$ & $\begin{array}{l}\text { Month } 6(W T), \text { month } 24 \\
(W T) \text { (6 replicates) }\end{array}$ \\
\hline
\end{tabular}

Table S1: Description of the RNA-seq dataset used in the study. (Contd. next page.)

${ }^{1}$ Triplicate except mentioned otherwise.

${ }^{2}$ Not included in the analysis due to low coverage (bellow $1 \mathrm{X}$ genome coverage or $29 \mathrm{M}$ sequenced reads for $R$. norvegicus ; genome coverage calculated using Lander-Waterman formula) 


\begin{tabular}{|c|c|c|c|c|}
\hline Species & Tissue & $\begin{array}{c}\text { Enrichment } \\
\text { protocol }\end{array}$ & $\begin{array}{l}\quad \begin{array}{l}\text { Sequencing } \\
\text { parameters }\end{array} \\
\text { Paired-end/single- } \\
\text { end, read length in } \\
\text { base pairs (bp), } \\
\text { millions of reads (M) }\end{array}$ & $\begin{array}{c}\text { Time points }{ }^{3} \text { and } \\
\text { conditions }\end{array}$ \\
\hline \multirow[t]{7}{*}{$\begin{array}{c}H . \\
\text { sapiens }\end{array}$} & $\begin{array}{l}\text { Fetal lungs } \\
\text { (IMR90) }\end{array}$ & Nascent RNA & $\begin{array}{l}\text { Paired-end, } 75 b p, 25 \\
\text { M }\end{array}$ & $\begin{array}{l}\text { Early passage, } \\
\text { late passage ( } 2 \\
\text { replicates) }\end{array}$ \\
\hline & $\begin{array}{l}\text { Fetal lungs } \\
\text { (IMR90) }\end{array}$ & $\begin{array}{c}\text { TruSeq Stranded } \\
\text { Total RNA } \\
\text { Library }\end{array}$ & $\begin{array}{l}\text { Paired-end, } 75 \text { bp, } 50 \\
\text { M }\end{array}$ & $\begin{array}{l}\text { Early passage, } \\
\text { late passage ( } 2 \\
\text { replicates) }\end{array}$ \\
\hline & $\begin{array}{l}\text { Umbilical vein } \\
\text { endothelial } \\
\text { (HUVECs) }\end{array}$ & Nascent RNA & $\begin{array}{l}\text { Paired-end, } 75 \text { bp, } 50 \\
\text { M }\end{array}$ & $\begin{array}{l}\text { Early passage, } \\
\text { late passage ( } 2 \\
\text { replicates) }\end{array}$ \\
\hline & $\begin{array}{l}\text { Umbilical vein } \\
\text { endothelial } \\
\text { (HUVECs) }\end{array}$ & $\begin{array}{c}\text { TruSeq Stranded } \\
\text { Total RNA } \\
\text { Library }\end{array}$ & $\begin{array}{l}\text { Paired-end, } 75 \text { bp, } \\
100 \mathrm{M}\end{array}$ & $\begin{array}{l}\text { Early passage, } \\
\text { late passage }\end{array}$ \\
\hline & \multirow[t]{2}{*}{$\begin{array}{c}\text { Skin } \\
\text { fibroblasts }\end{array}$} & \multirow[t]{2}{*}{$\begin{array}{c}\text { TruSeq Stranded } \\
\text { Total RNA } \\
\text { Library }\end{array}$} & $\begin{array}{l}\text { Paired-end, } 100 \text { bp, } \\
100 \mathrm{M}\end{array}$ & $\begin{array}{l}\text { Progeria patient } \\
\text { (HSS) ( } 2 \\
\text { replicates) }\end{array}$ \\
\hline & & & $\begin{array}{l}\text { Paired-end, } 75 \text { bp, } \\
100 \mathrm{M}\end{array}$ & $\begin{array}{l}\text { Healthy donor, } \\
\text { sex/age matched } \\
\text { with progeria } \\
\text { patient ( } 2 \\
\text { replicates) }\end{array}$ \\
\hline & Blood & $\begin{array}{l}\text { TruSeq Stranded } \\
\text { Total RNA } \\
\text { Library }\end{array}$ & $\begin{array}{l}\text { Paired-end, } 75 \text { bp, } 70 \\
\text { M }\end{array}$ & $\begin{array}{l}\text { Healthy donor, } 6 \\
\text { females, } 6 \text { males, } \\
\text { age range: } 21-70\end{array}$ \\
\hline
\end{tabular}

Table S1: Description of the RNA-seq dataset used in the study. (Contd. from previous page.)

\footnotetext{
${ }^{3}$ Triplicate except mentioned otherwise.
} 